\title{
REUTILIZACIÓN DE AGUAS DEPURADAS EN RIEGO
}

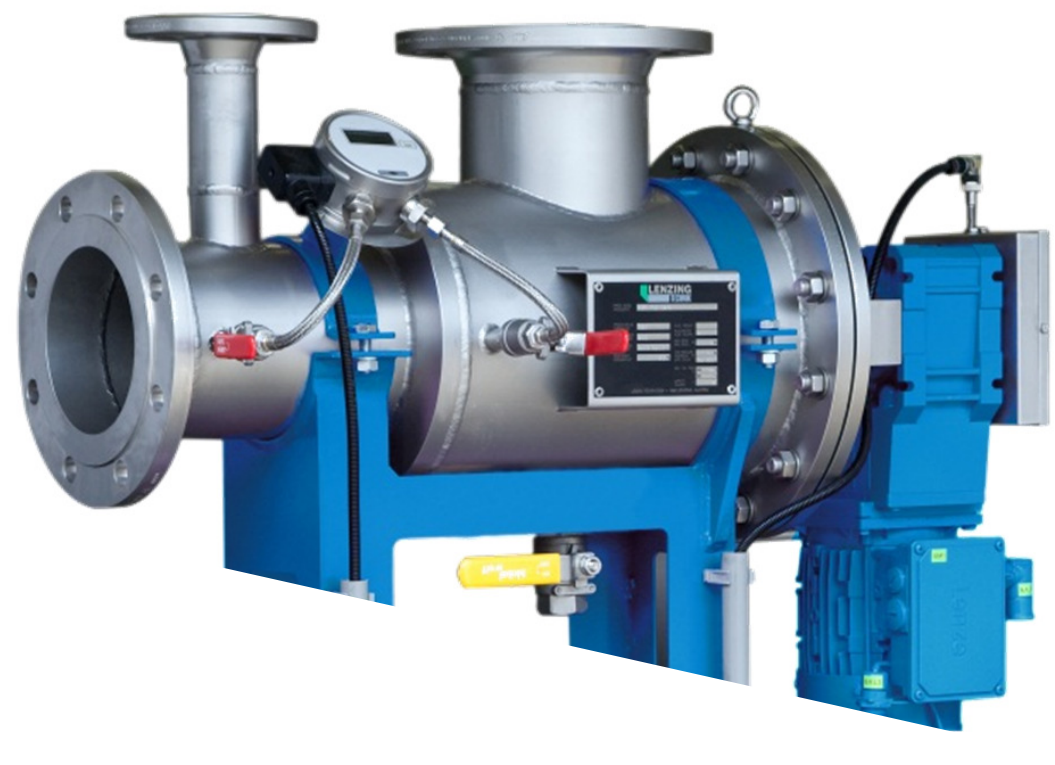

$$
\begin{aligned}
& \text { HAWLE-OPTIFIL } \\
& \text { HAWLE-CANFIL } \\
& \text { The new spirit of filtration }
\end{aligned}
$$

HAWLE. MADE FOR GENERATIONS. 


\section{RESUMEN}

\section{Visión general del filtrado}

\section{HAWLE-OPTIFIL}

»Visión general de la reutilización

» Simulación de funcionamiento

»Detalles de construcción

»Certificados

»Especificaciones Técnicas

》Referencias

»Planta Móvil de tratamiento de agua

\section{HAWLE-CANFIL}

»Áreas de aplicación

» Funcionamiento

»Dimensiones

»Especificaciones Técnicas

»Referencias 


\section{Visión general del filtrado}

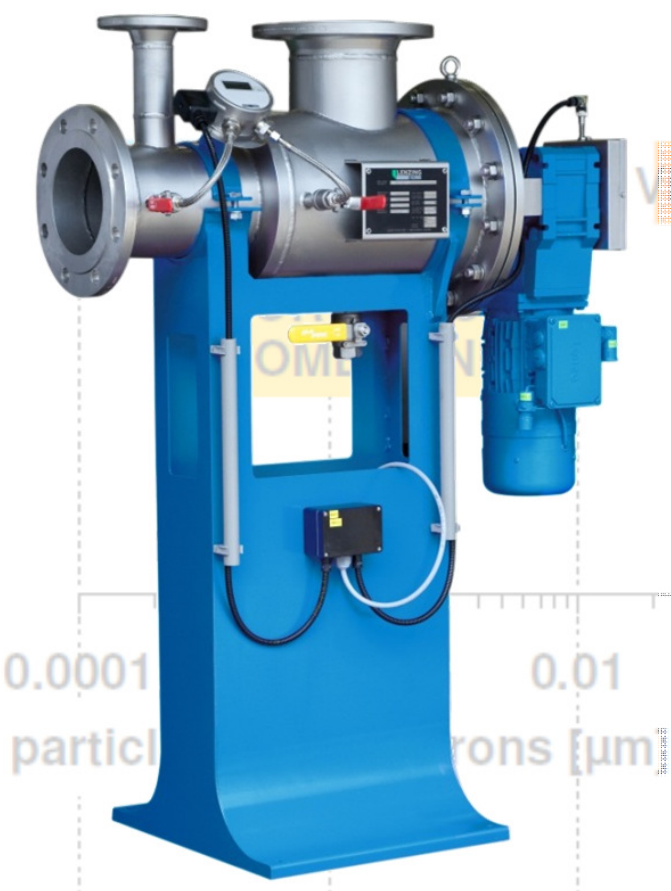

HAWLE-OPTIFIL

Tamaño de partículas $>0.001 \mathrm{~mm}=1 \mu \mathrm{m}$ !!!

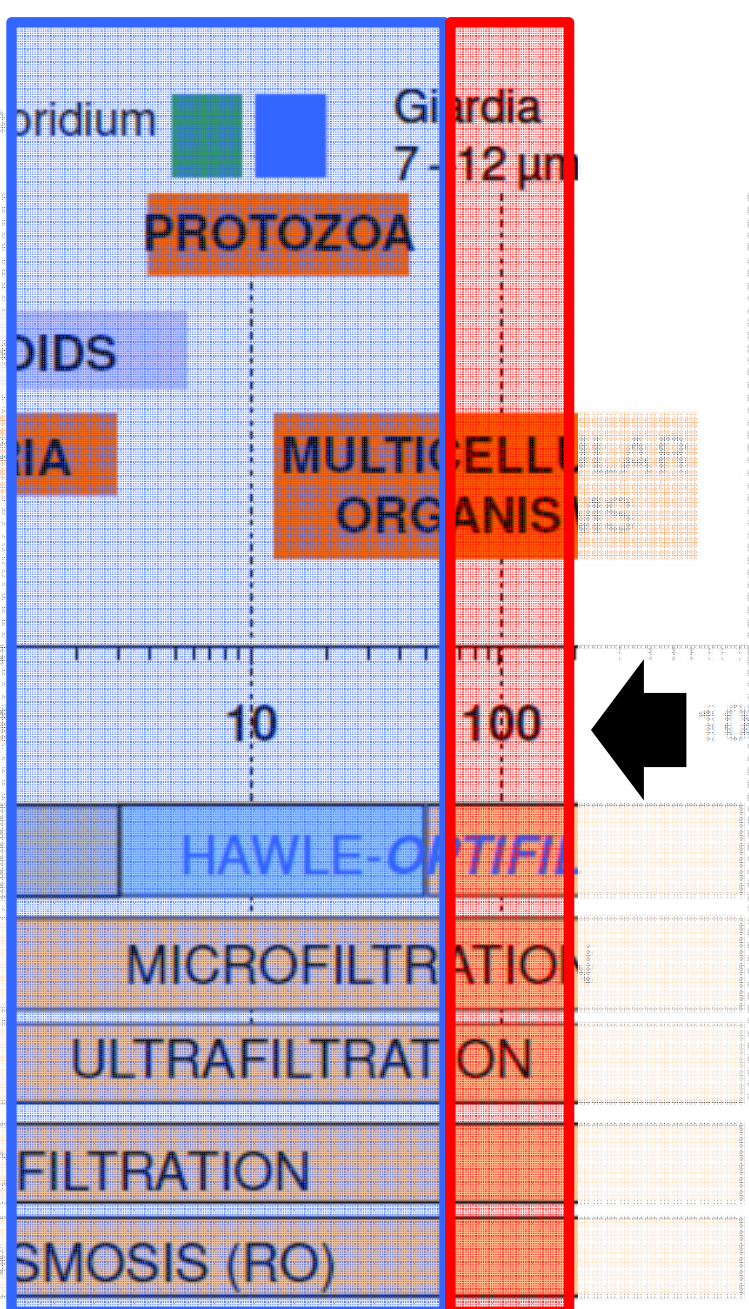

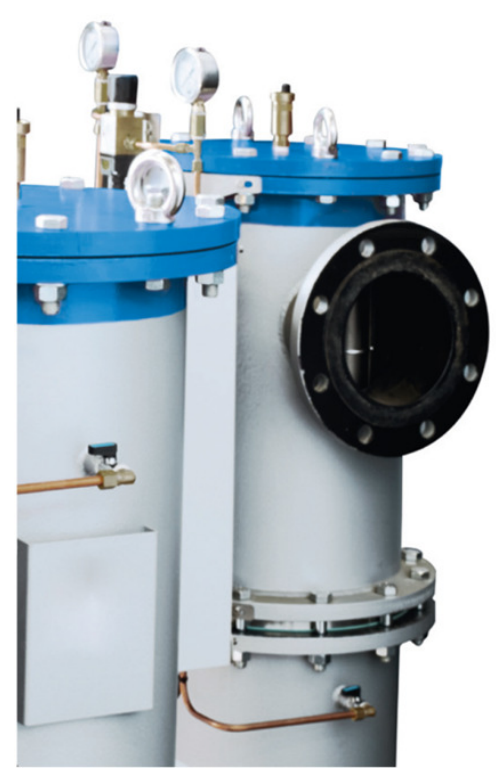

HAWLE-CANFIL

Tamaño de partículas $>0.05 \mathrm{~mm}=50 \mu \mathrm{m}$ !!! 


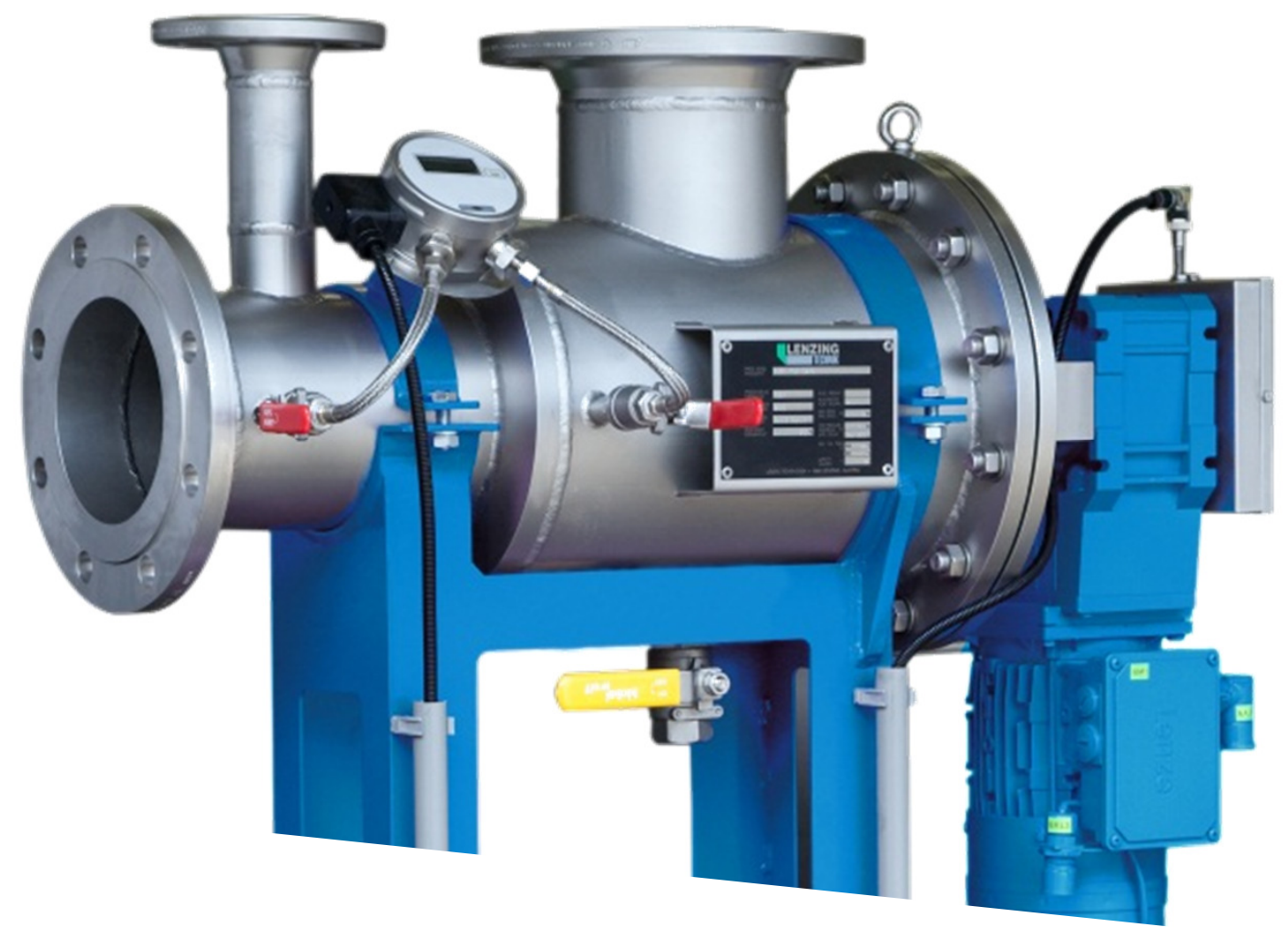

The new spirit of filtration 


\section{Visión general de la reutilización}

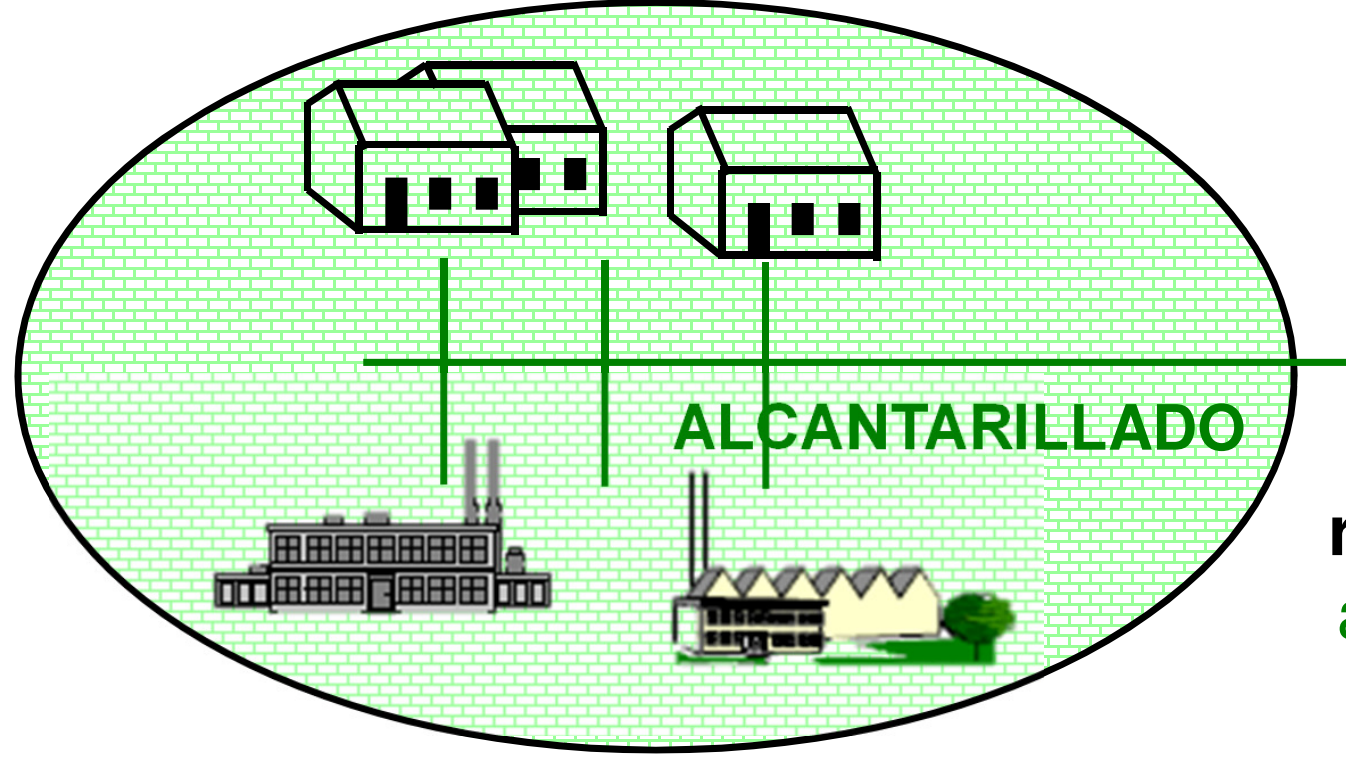

REUTILIZACIÓN EN REGADÍO

Real Decreto 1620/2007: Calidad 2.1

$><1$ huevo vivo de nematodo / 10 I

$><100$ U.F.C. $/ 100 \mathrm{ml}$

> Solidos en suspensión < 20 mg/l

$>$ Turbidez < 10 NTU

(*) Unidades Formadoras de Colonias

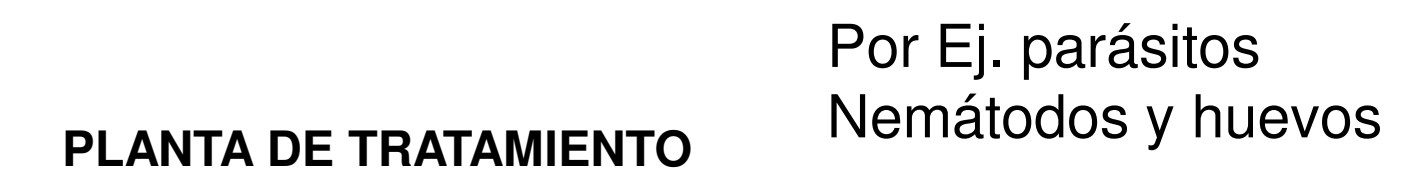
DE AGUA RESIDUAL

Nemátodos y huevos

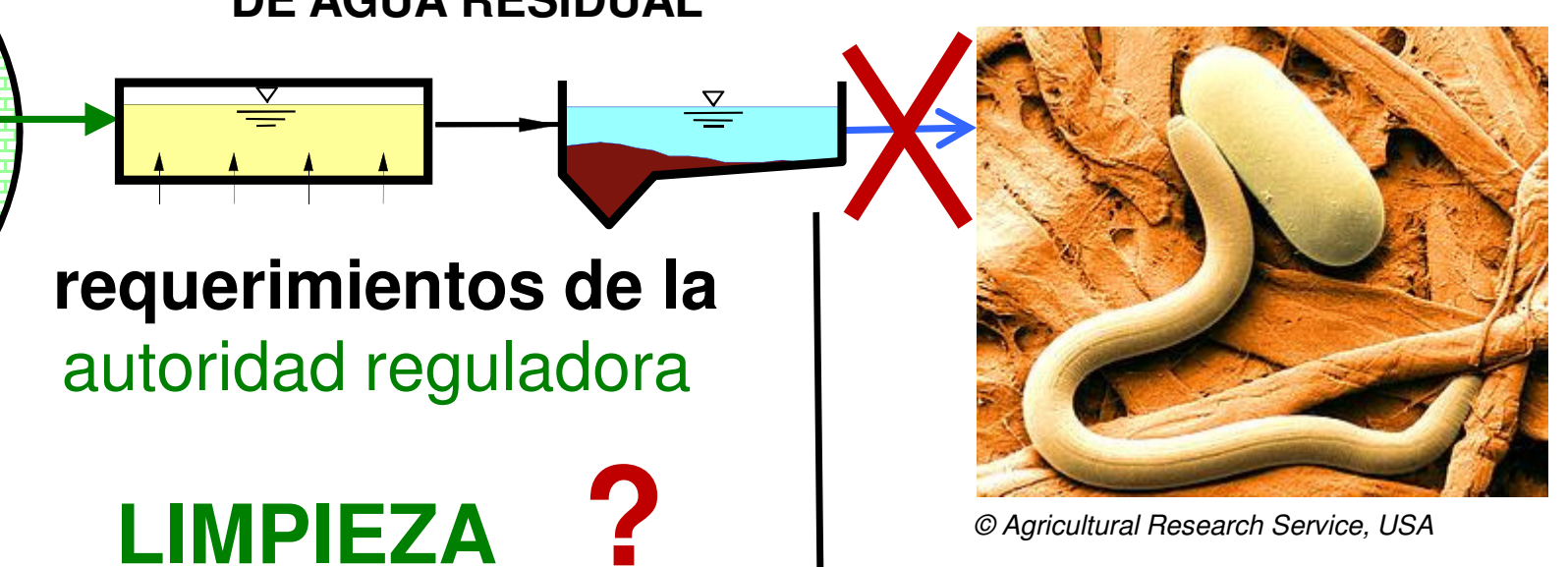

LIMPIEZA ?

(c) Agricultural Research Service, USA

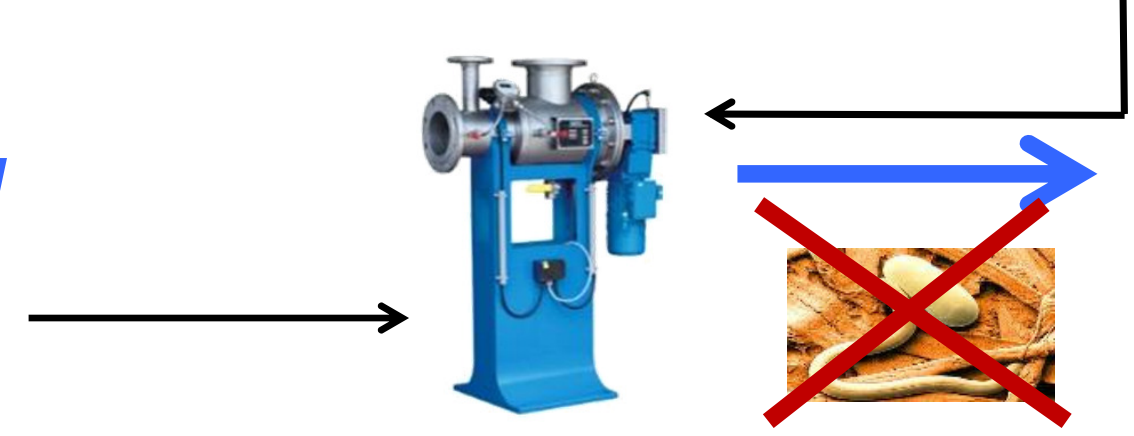

HAWLE-OPTIFIL

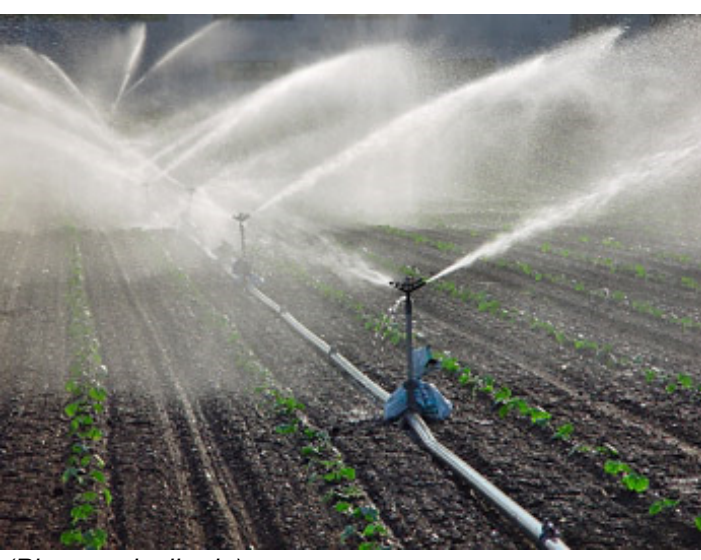

HAWLE. MADE FOR GENERATIONS. 


\section{Detalle HAWLE-OPTIFIL}
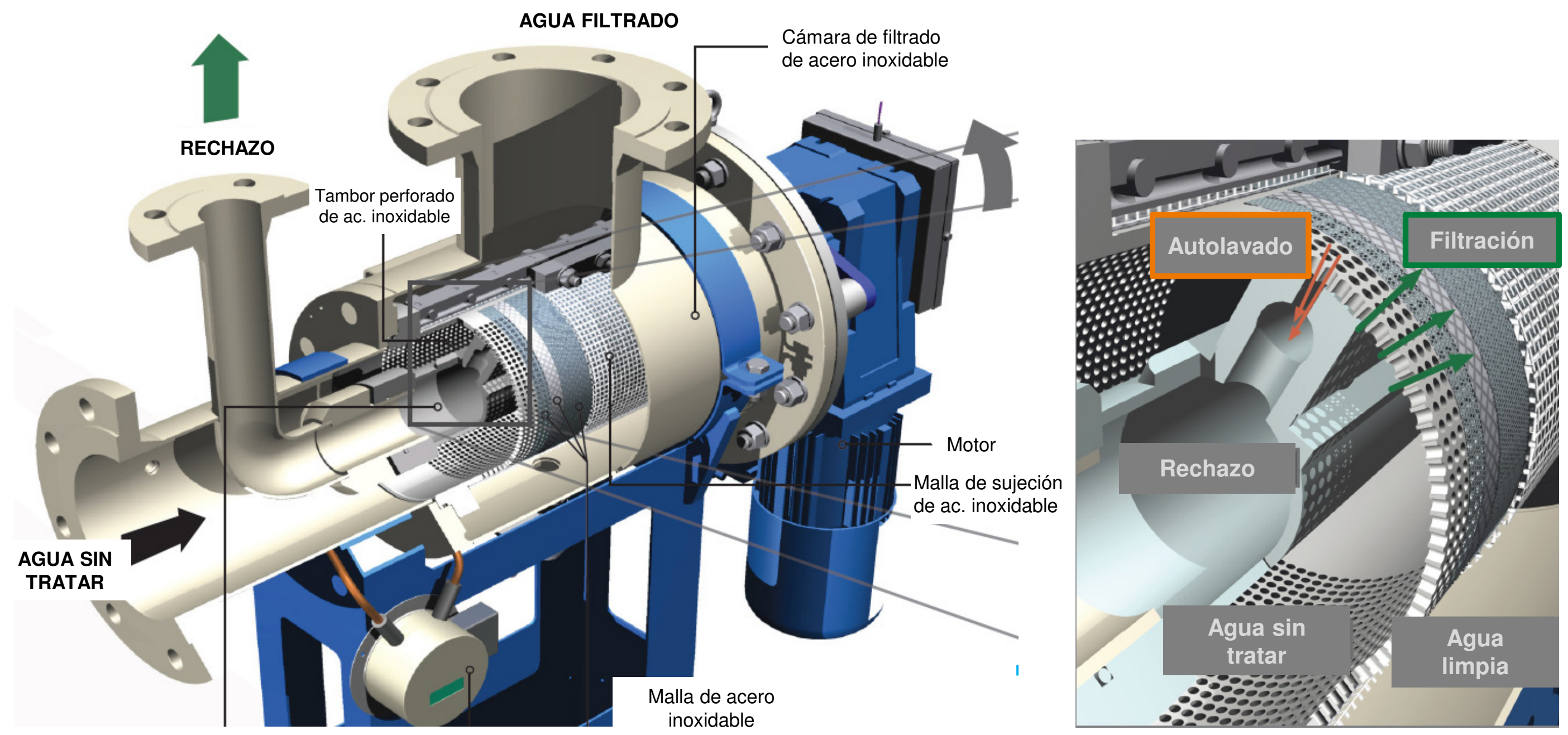

Dispositivo de autolavado
Sensor de presión diferencial

HAWLE. MADE FOR GENERATIONS. 


\section{Detalle HAWLE-OPTIFIL}

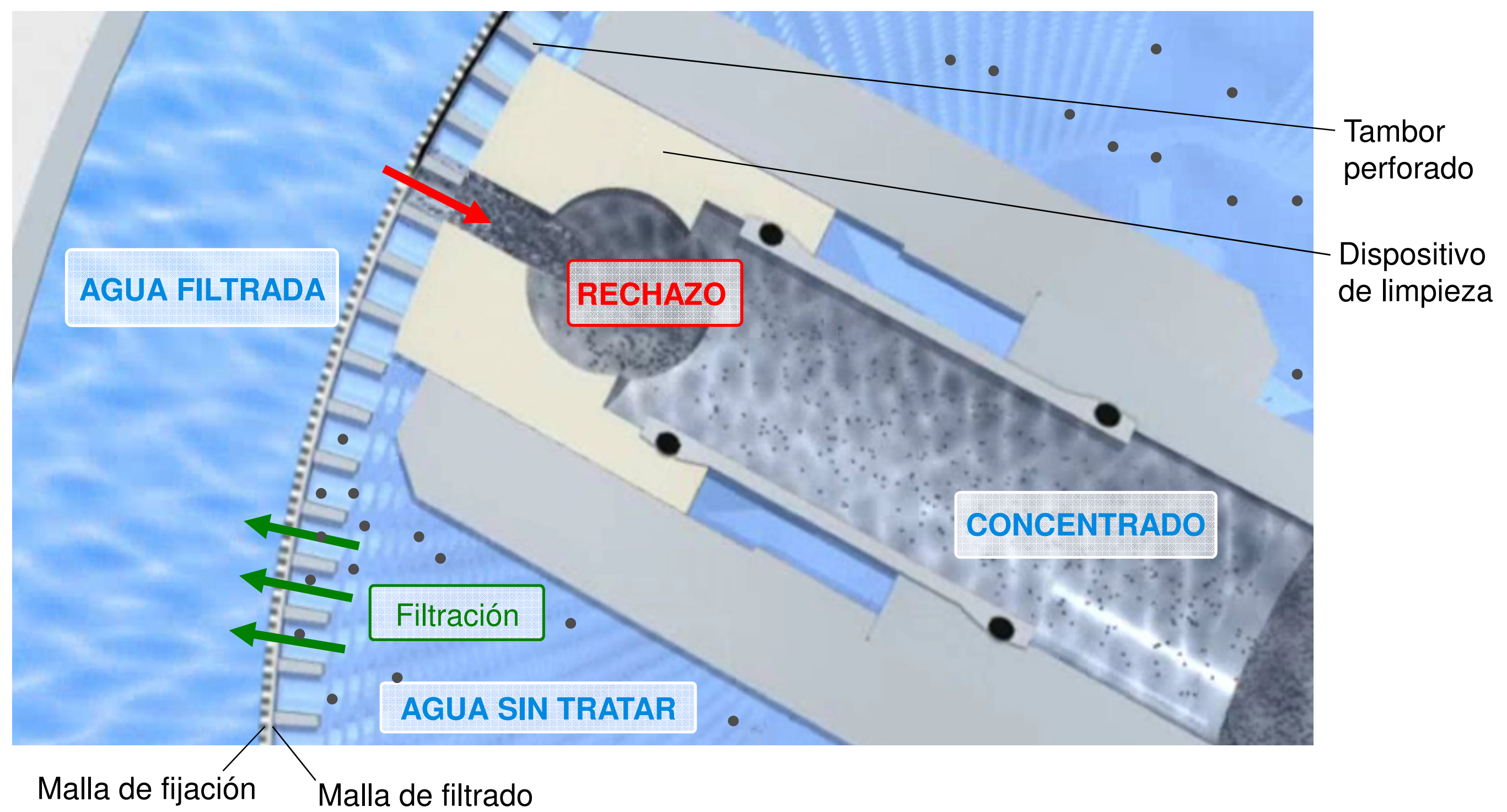




\section{Detalle HAWLE-OPTIFIL}

- Tipos de Malla disponibles

» $50 \mu \mathrm{m}$ - Acero Inoxidable 316

» $25 \mu \mathrm{m}$ - Acero Inoxidable 316

»10 $\mu \mathrm{m}$ - Acero Inoxidable 316

» $6 \mu \mathrm{m}$ - Poliamida (Agua salada)

» 5,5 $\mu \mathrm{m}$ - Acero Inoxidable recubierto con PFA (teflón)

» $3 \mu \mathrm{m}$ - Fibras comprimidas (fieltro)

» $2 \mu \mathrm{m}$ - Acero Inoxidable 316

» $1 \mu \mathrm{m}$ - Acero Inoxidable 316

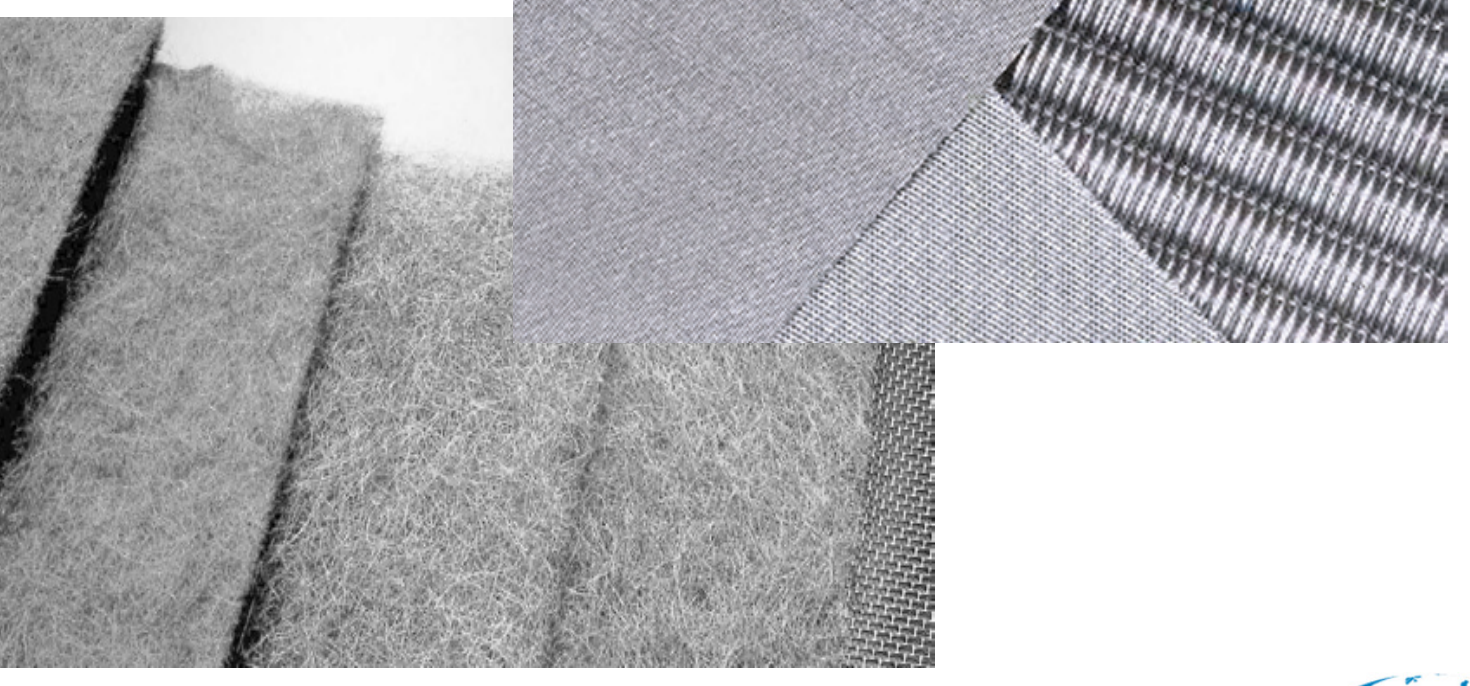




\section{Detalle HAWLE-OPTIFIL}

- Modelos de apertura rápida
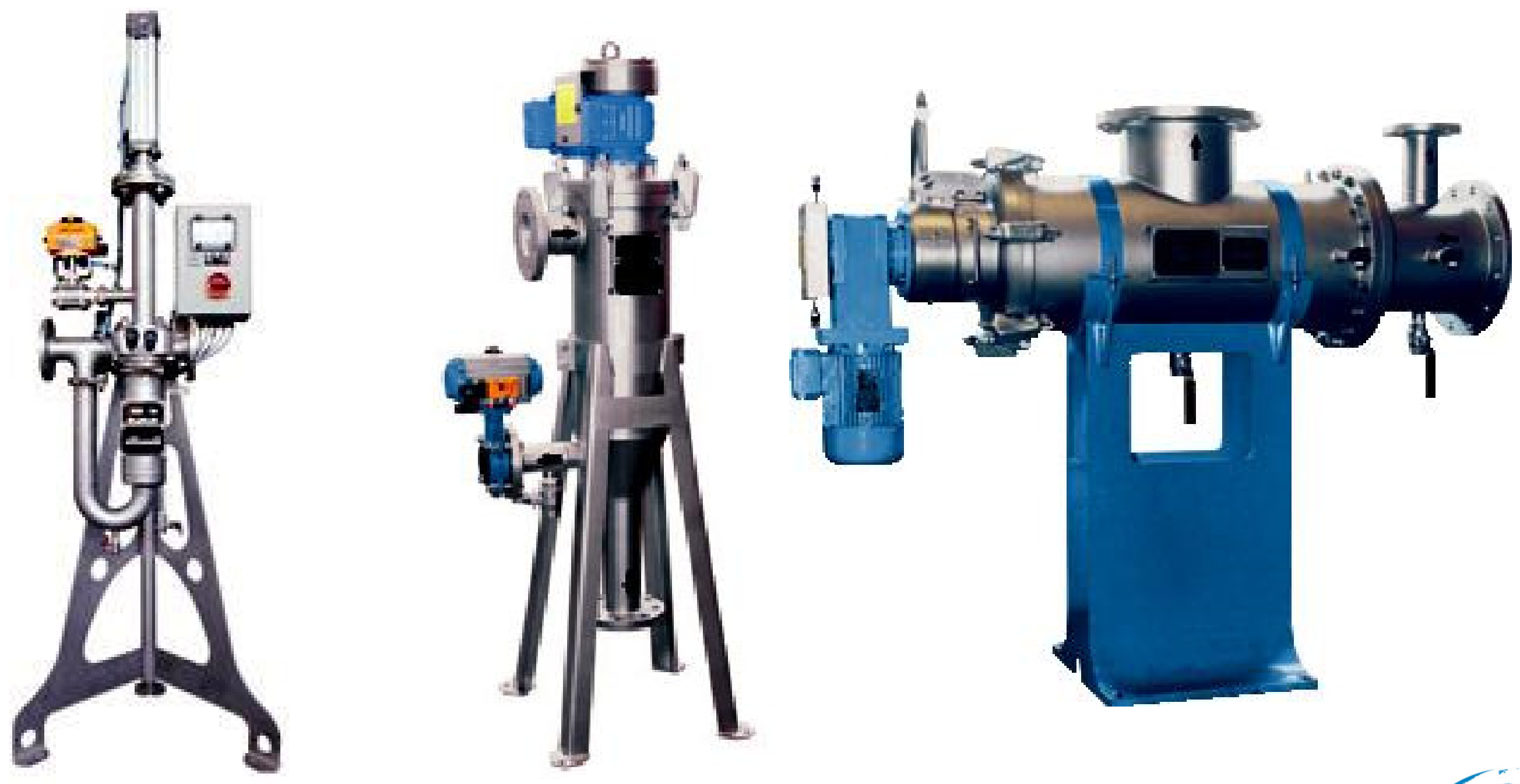

HAWLE. MADE FOR GENERATIONS. 


\section{Certificados HAWLE-OPTIFIL}

- Certificado para agua potable (ÖVGW)

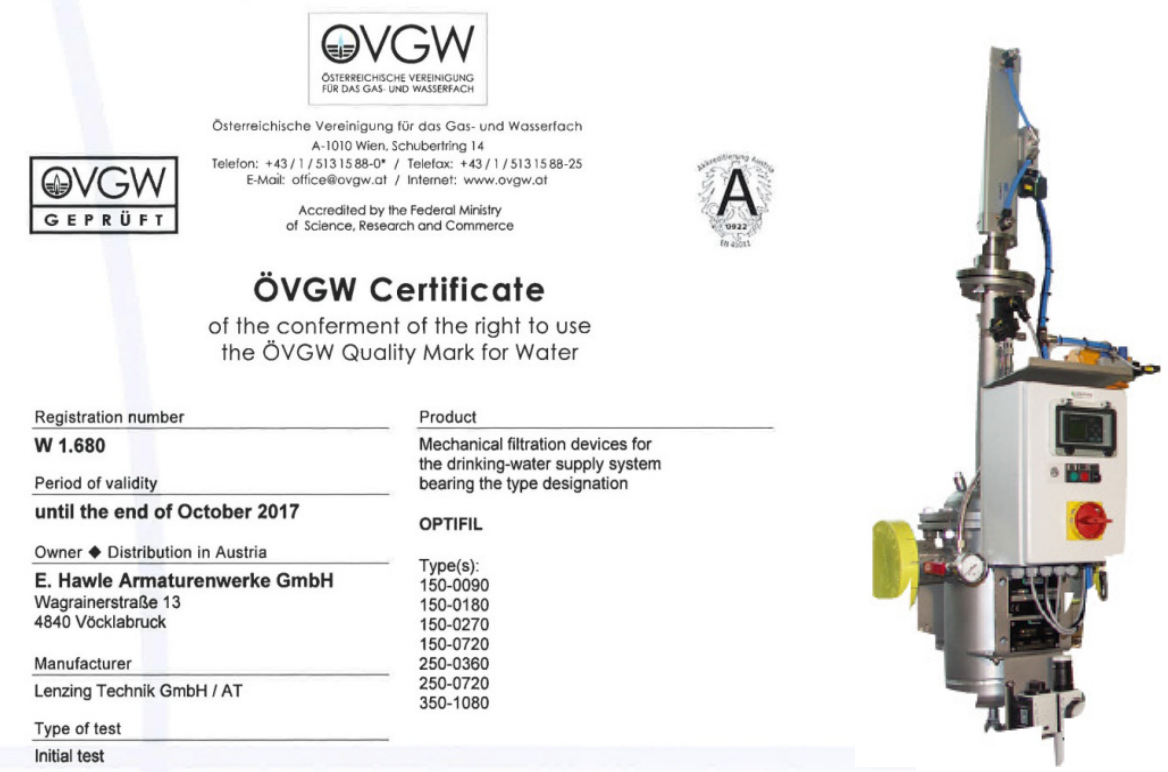

- Está certificado el funcionamiento del filtro y el grado de filtración.

$\frac{\text { Test report }}{412.320 \text { dated } 2 \text { September } 2014}$

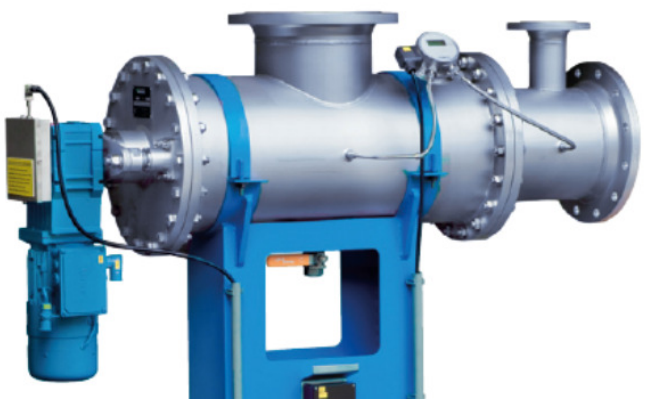

- Primer Certificado de un filtro automático por retrolavado en todo el mundo.

Quality standards / Testing directives

- QS-W807 (version December 2013)

» Número de registro ÖVGW: W 1.680 


\section{Esp. Técnicas HAWLE-OPTIFIL}

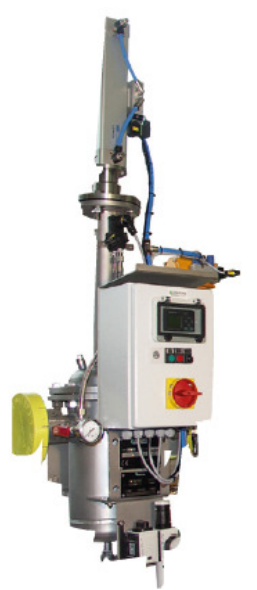

DN 50

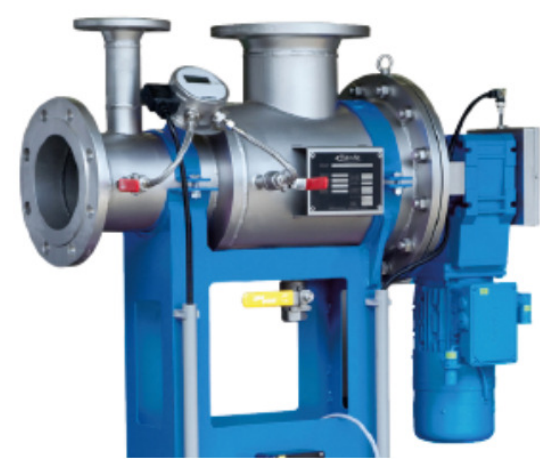

DN 150

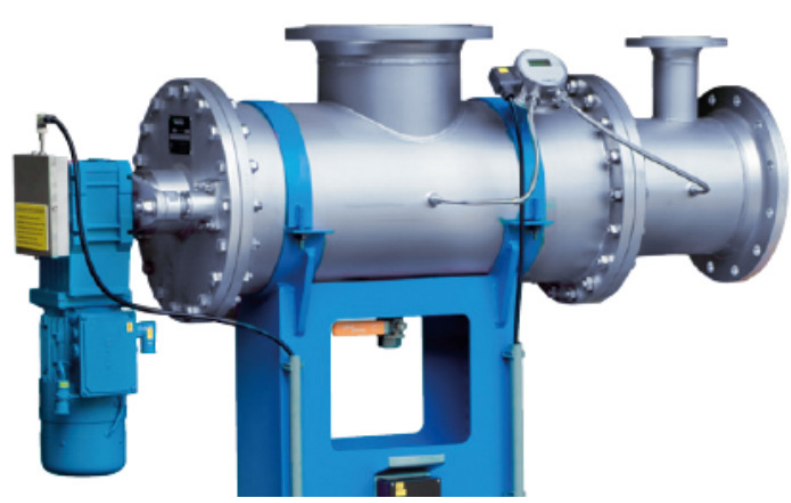

DN 250 / 350

\begin{tabular}{|c|c|c|c|c|c|c|c|c|}
\hline Modelo & $\begin{array}{c}\text { Presión } \\
\text { de trabajo } \\
\text { [bar] }\end{array}$ & $\begin{array}{c}\text { DN } \\
\text { Entrada y } \\
\text { salida } \\
\text { [mm/pulg.] }\end{array}$ & $\begin{array}{l}\text { DN } \\
\text { Salida de } \\
\text { limpieza } \\
\text { [mm/pulg.] }\end{array}$ & $\begin{array}{c}\text { Área de } \\
\text { ffltrado } \\
\left.\qquad \mathrm{cm}^{2}\right]\end{array}$ & $\begin{array}{c}\text { Caudal } \\
\text { (3 pm) } \\
{\left[m^{3 / h}\right]}\end{array}$ & $\begin{array}{c}\text { Caudal } \\
\text { (25 um) } \\
{\left[\mathrm{m}^{3} / \mathrm{h}\right]}\end{array}$ & 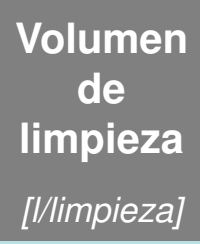 & $\begin{array}{c}\text { Duración } \\
\text { de la } \\
\text { limpieza } \\
\text { [seg/limpieza] }\end{array}$ \\
\hline 050-0200 & $10 / 16$ & 50 / 2" & 25 / 1" & 140 & 4.8 & 20 & 1.1 & 2 \\
\hline $100-0270$ & $10 / 16$ & 80 / 3" & 50 / 2" & 200 & 7 & 30 & 1.4 & 3 \\
\hline $150-0270$ & 10 & 150 / 6" & 50 / 2" & 570 & 19 & 100 & 4.3 & 3 \\
\hline $150-0720$ & 10 & 150 / 6" & 80 / 3" & 1520 & 50 & 140 & 5.6 & 3 \\
\hline $250-0720$ & 10 & 250 / 10" & 80 / 3" & 2500 & 85 & 380 & 19.0 & 4 \\
\hline $350-1080$ & 10 & 350 / 14" & 100 / 4" & 4800 & 163 & 600 & 36.5 & 5 \\
\hline
\end{tabular}

HAWLE. MADE FOR GENERATIONS. 


\section{Dimensiones HAWLE-OPTIFIL}

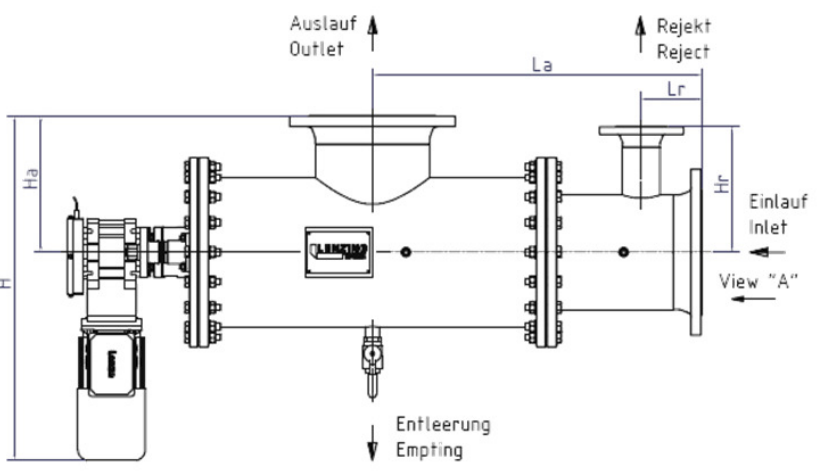

DN $250 / 350$

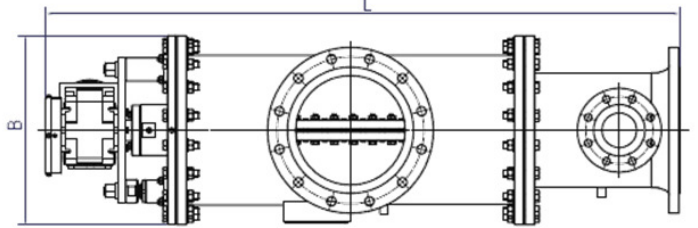

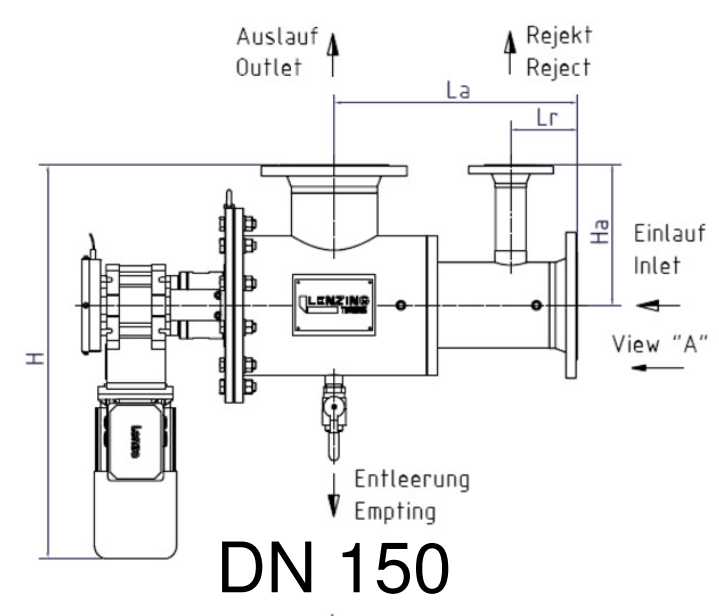

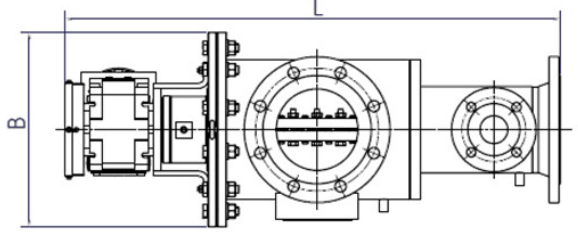

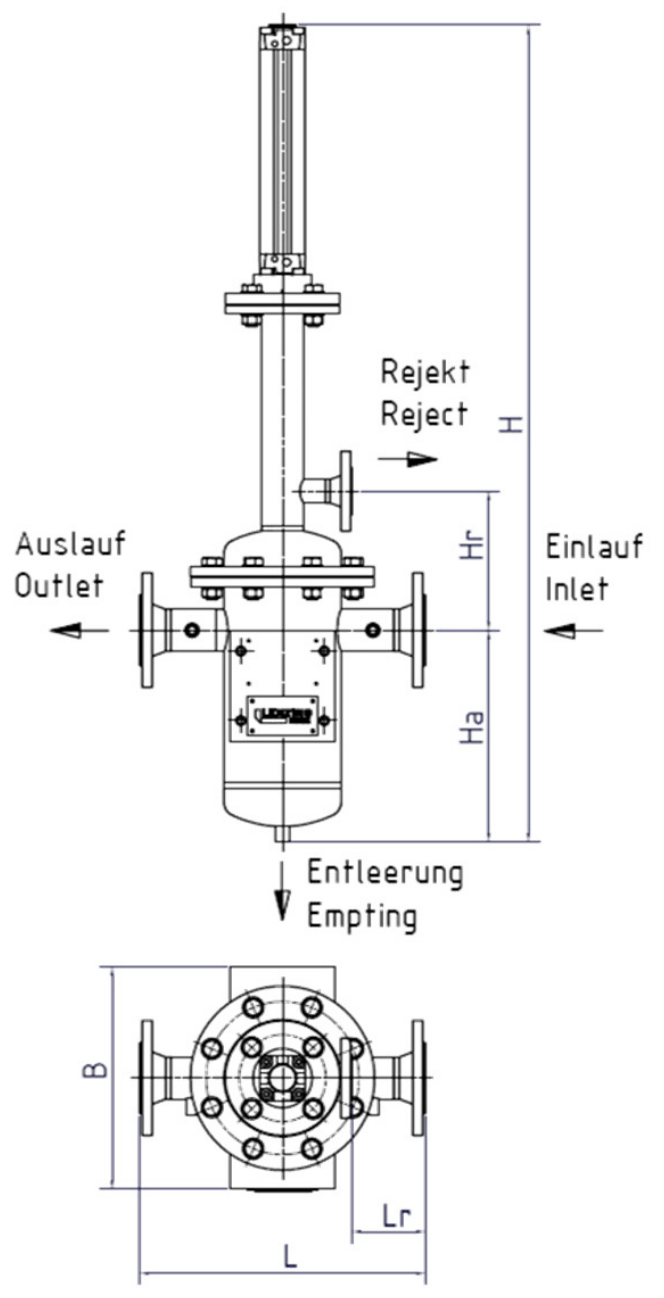

DN 50

HAWLE. MADE FOR GENERATIONS.

\begin{tabular}{|c|c|c|c|c|}
\hline Modelo & L & B & H & $\begin{array}{c}\text { Peso } \\
{[\mathrm{mm}]}\end{array}$ \\
\hline $050-0200$ & 488 & 410 & 1394 & 80 \\
\hline $100-0270$ & 512 & 644 & 1451 & 150 \\
\hline $150-0270$ & 967 & 490 & 733 & 207 \\
\hline $150-0720$ & 1400 & 490 & 733 & 243 \\
\hline $250-0720$ & 1500 & 530 & 820 & 317 \\
\hline $350-1080$ & 1870 & 630 & 820 & 461 \\
\hline
\end{tabular}




\section{Dimensiones HAWLE-OPTIFIL}
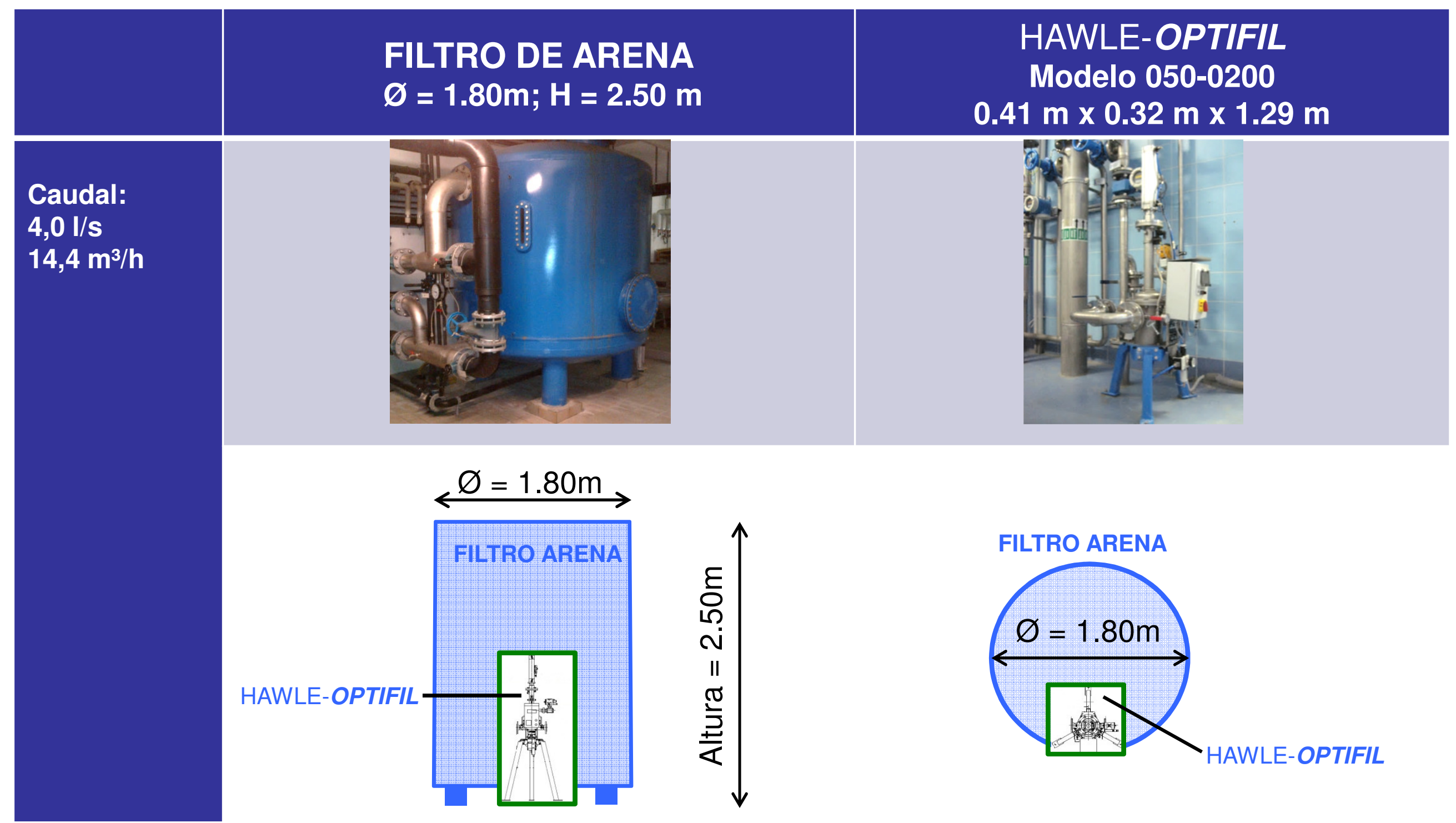

HAWLE. MADE FOR GENERATIONS. 


\section{Referencias HAWLE-OPTIFIL}

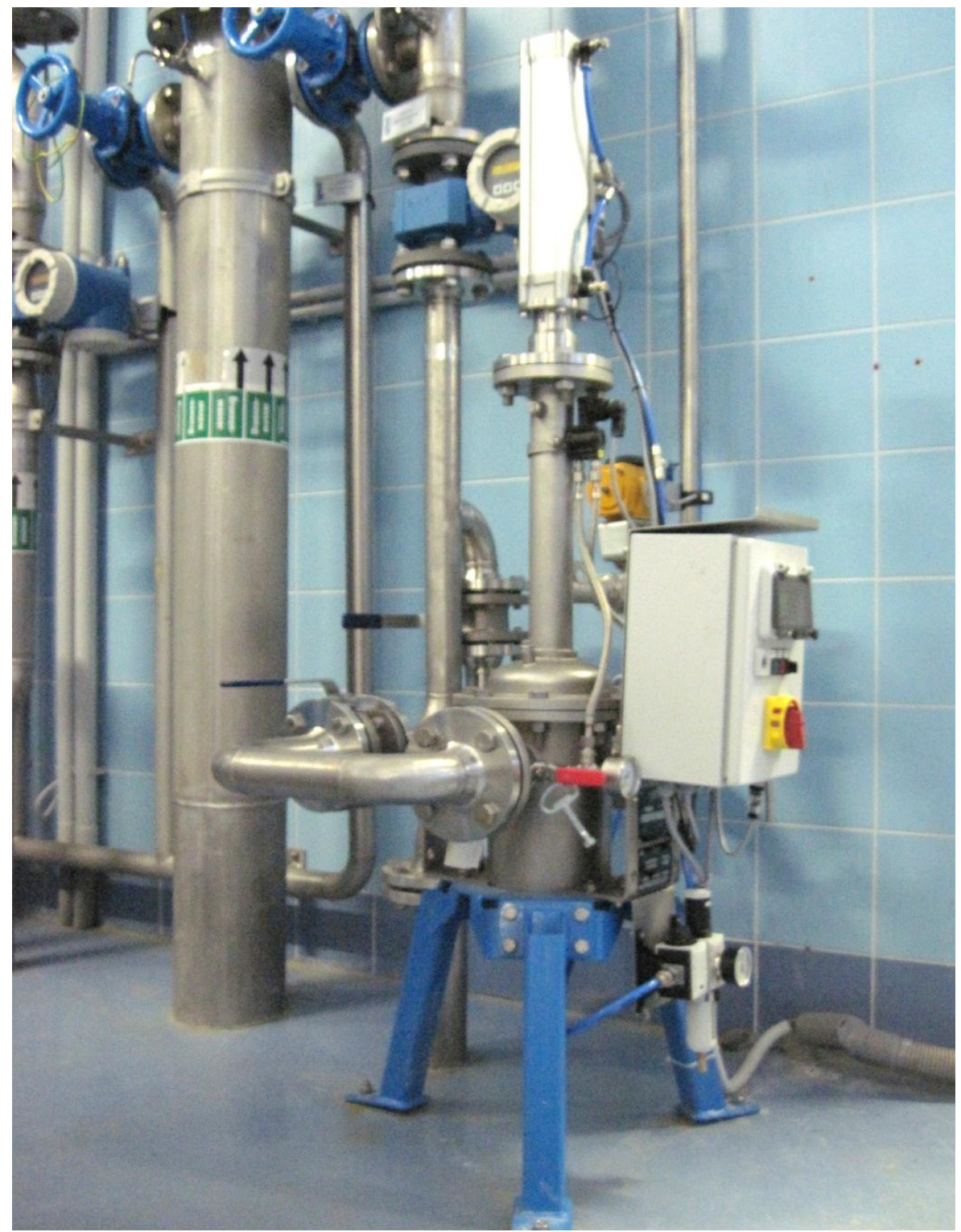

\begin{tabular}{|c|c|}
\hline Planta & Krottendorf DN 50 Asociación de aguas Unteres Lafnitztal \\
\hline Fuente agua sucia & pozo \\
\hline Caudal & $16 \mathrm{~m}^{3} / \mathrm{h}$ \\
\hline Objetivo & Eliminar partículas y reducir la turbidez (arena de cuarzo) \\
\hline Condiciones & $\begin{array}{l}\text { - Durante el arranque de la bomba una gran cantidad de } \\
\text { partículas es impulsada hacia el sistema } \\
\text { - Implementación en el sistema SCADA existente }\end{array}$ \\
\hline Solución & HAWLE-OPTIFIL con malla de $50 \mu \mathrm{m}$ de acero inoxidable \\
\hline
\end{tabular}




\section{Referencias HAWLE-OPTIFIL}

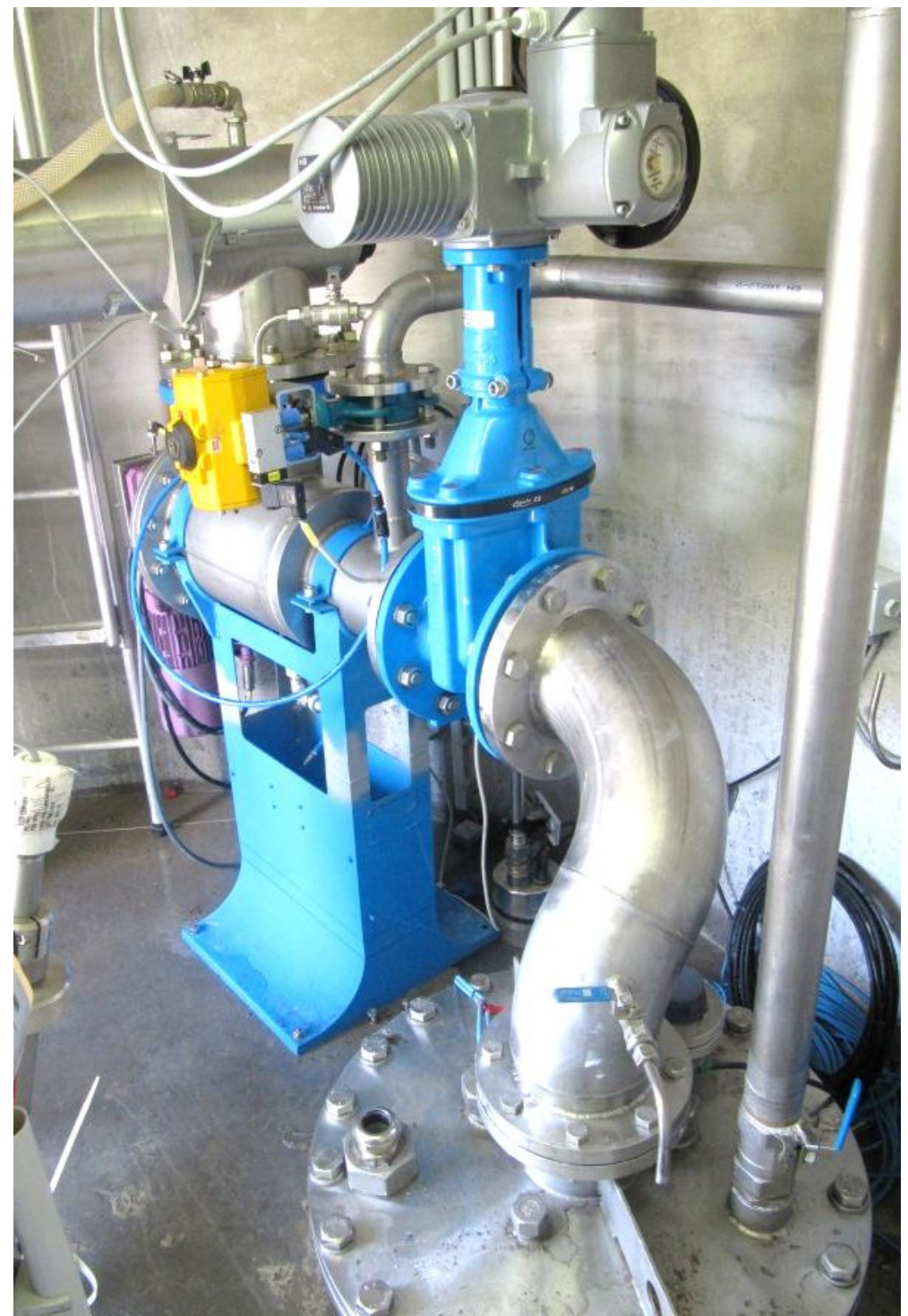

\begin{tabular}{|l|l|}
\hline Planta & Dobersdorf DN 150 Asociación de aguas Unteres \\
\hline Fuente agua & $\begin{array}{l}\text { Pozo de acuífero confinado } \\
57 \mathrm{~m}^{3} / \mathrm{h}\end{array}$ \\
\hline Caudal & $\begin{array}{l}\text { Eliminar partículas y reducir la turbidez (arena de cua } \\
\text { proteger el tratamiento UV }\end{array}$ \\
\hline Objetivo & $\begin{array}{l}\text { Espacio limitado para la instalación } \\
\text { - Implementación en el sistema SCADA existente }\end{array}$ \\
\hline Condiciones & HAWLE-OPTIFIL malla de 10 $10 \mathrm{~m}$ de acero inoxidable
\end{tabular}

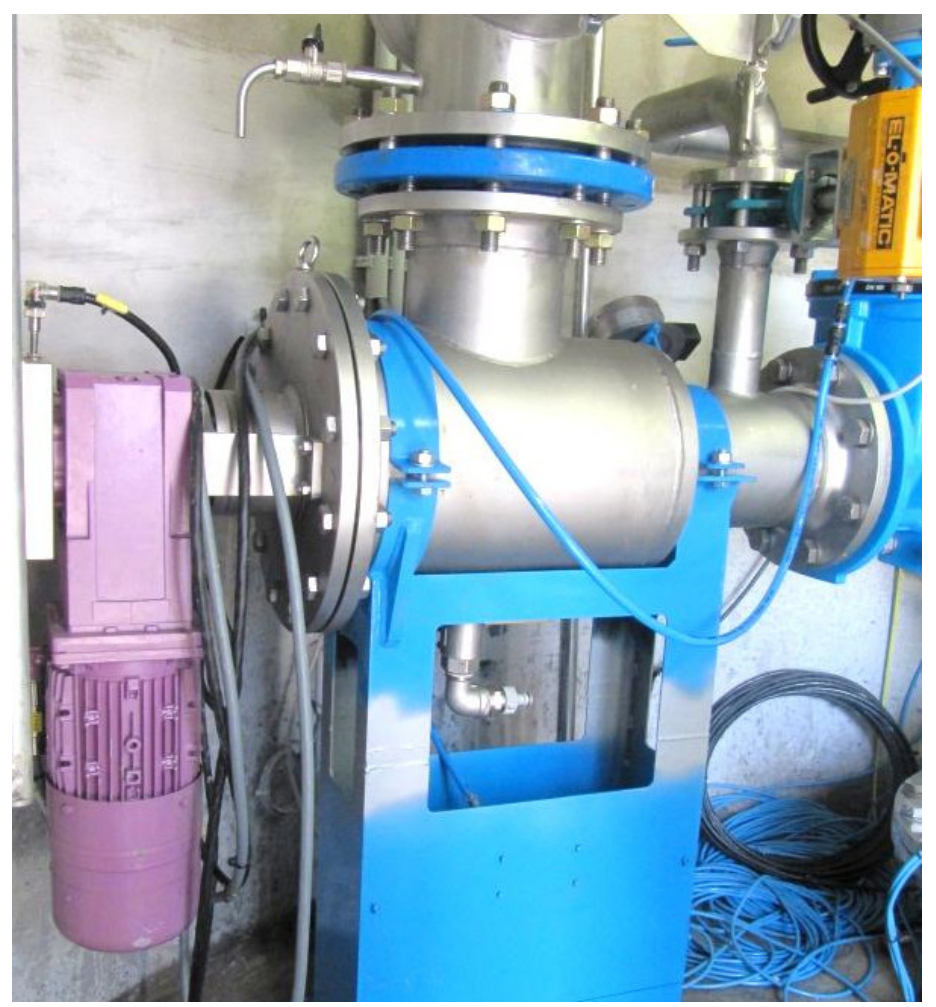




\section{Referencias HAWLE-OPTIFIL}

Ejemplo: Asociación de aguas "Unteres Lafnitztal"

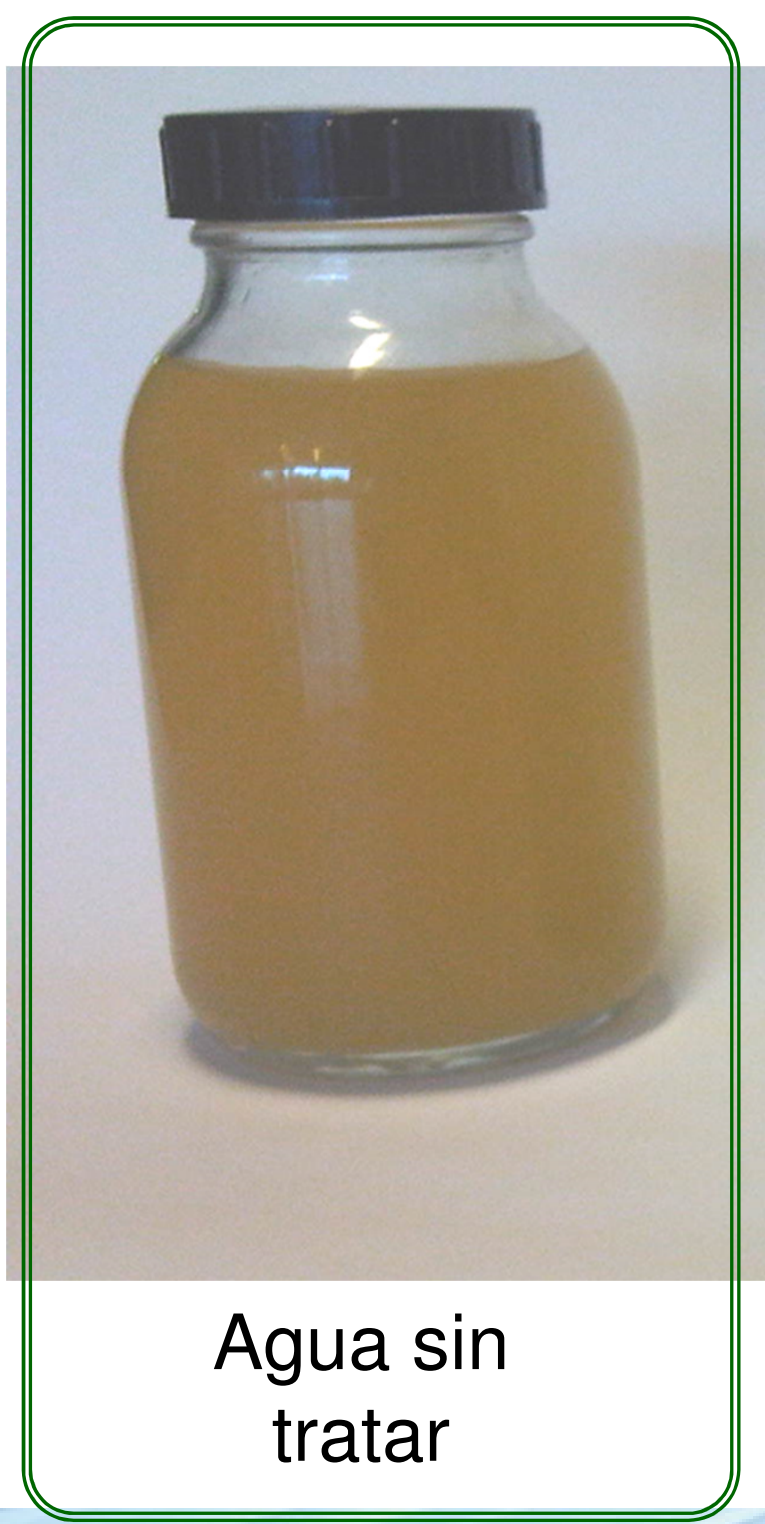

Filtrado;
Filtrado; malla de $50 \mu \mathrm{m}$
Ahora, el agua puede ser desinfectada también mediante rayos UV.

HAWLE. MADE FOR GENERATIONS. 


\section{Planta móvil de tratamiento de agua}
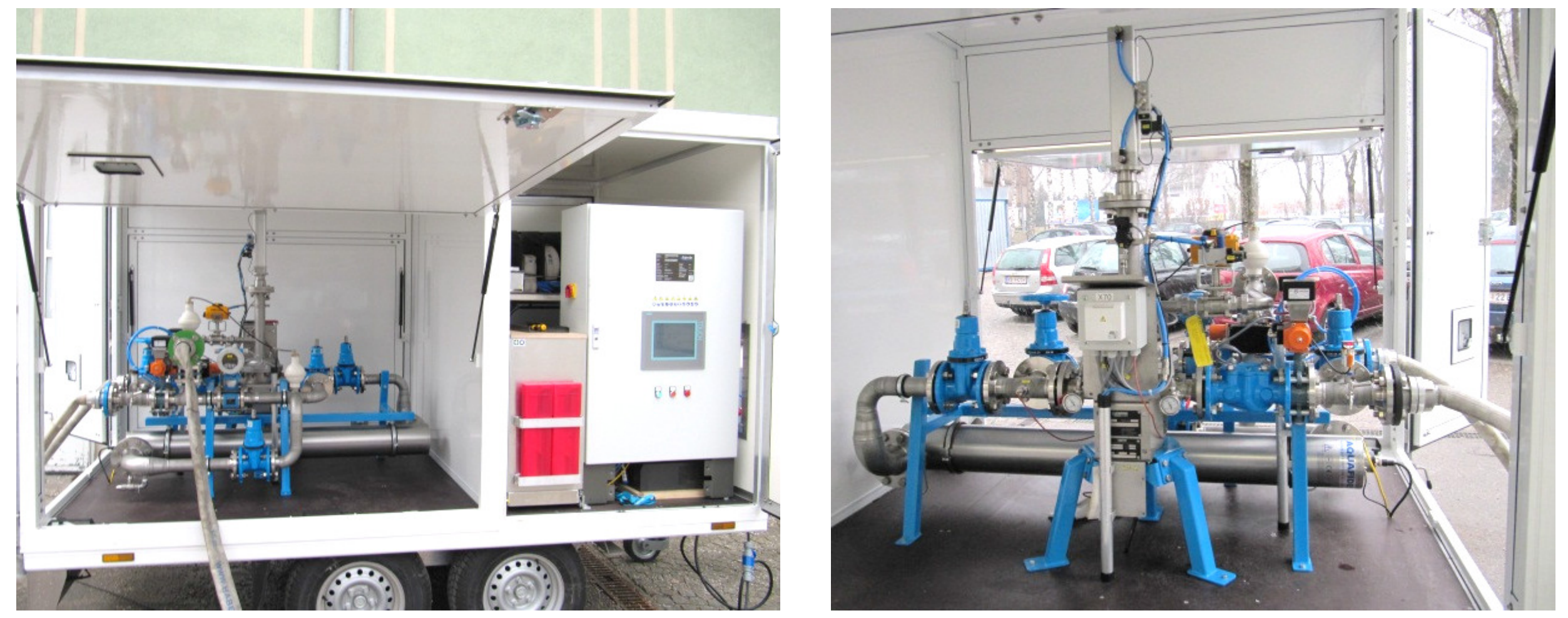

Remolque equipado con HAWLE-OPTIFIL DN 50 y tratamiento UV (opcional)

Funcionamiento:

Capacidad:

Aplicaciones:

Equipamiento:
- Eliminación de partículas, de turbidez y tratamiento UV

- De 5 a $20 \mathrm{~m}^{3} / \mathrm{h}$

- Distribución de emergencia de agua potable

- Ensayos piloto para los sistemas de filtrado

- Filtro autolimpiante automático HAWLE-OPTIFIL

- Tratamiento UV HAWLE-UV 


\section{Planta móvil de tratamiento de agua 1 hawlewater}

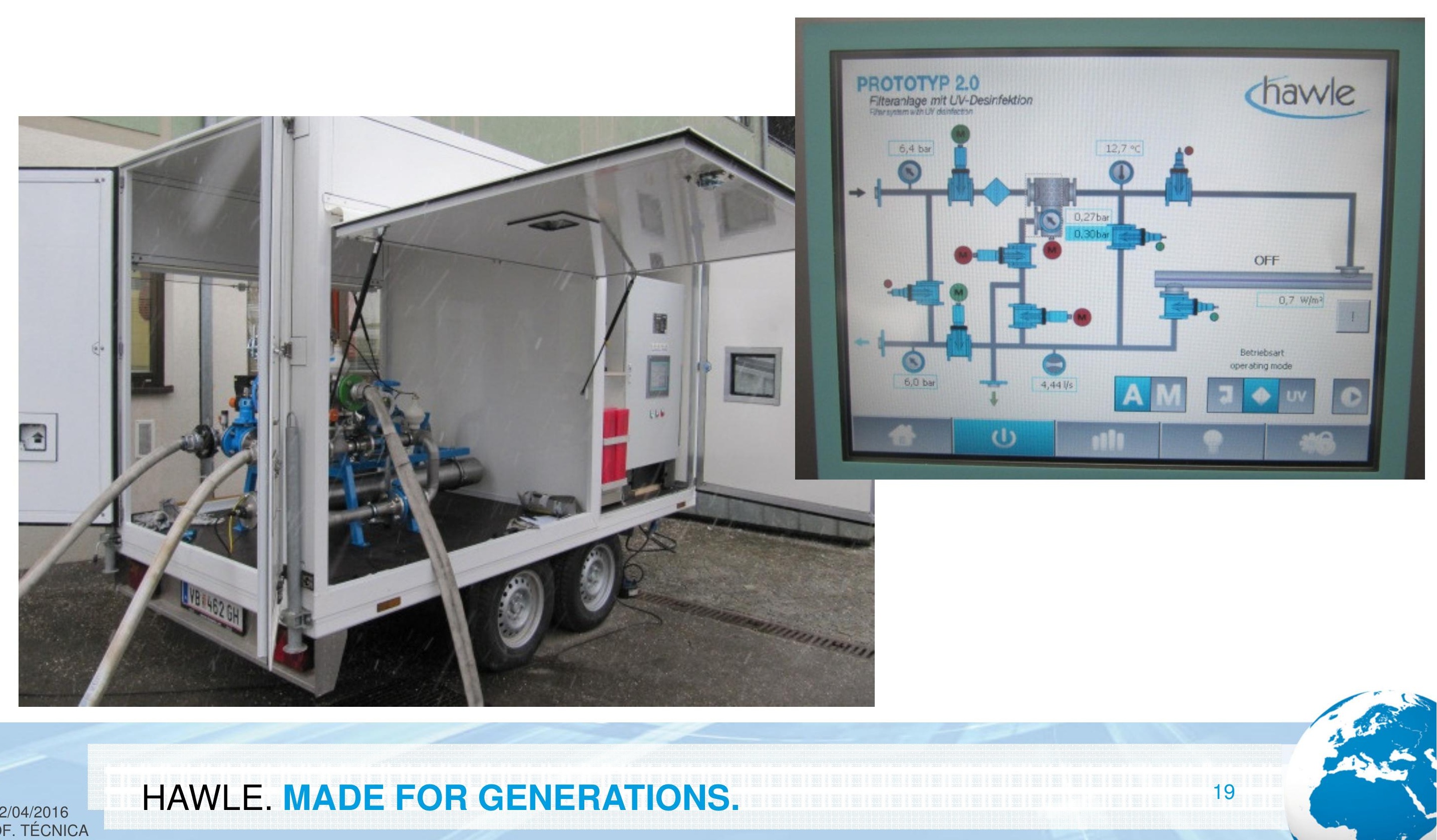


- Visión general de la reutilización

- Simulación de funcionamiento

- Detalles de construcción

- Certificados

- Especificaciones Técnicas

- Referencias

- Planta Móvil de tratamiento de agua 


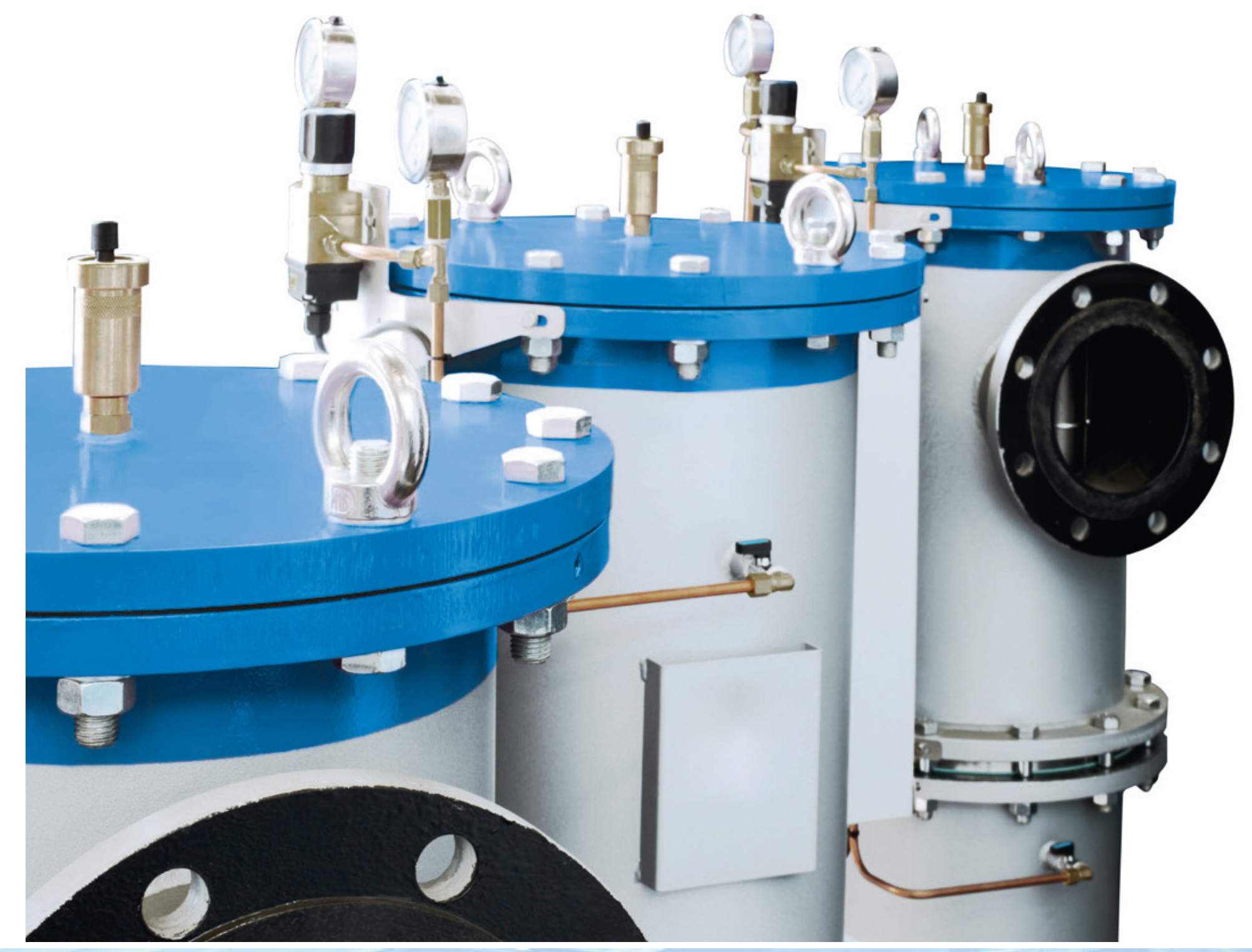




\section{HAWLE-CANFIL}

\section{ÁREAS DE APLICACIÓN}

- Filtrado de aguas para riego

- Tratamiento de aguas residuales

- Fabricación de nieve artificial

- Industria de celulosa y papel

- Fabricación de fibras

- Industria química y petroquímica

- Industria textil

- Industria siderúrgica

- Industria de la automoción

- Tratamiento de agua potable

- Plantas de producción de energía 


\section{Funcionamiento HAWLE-CANFIL

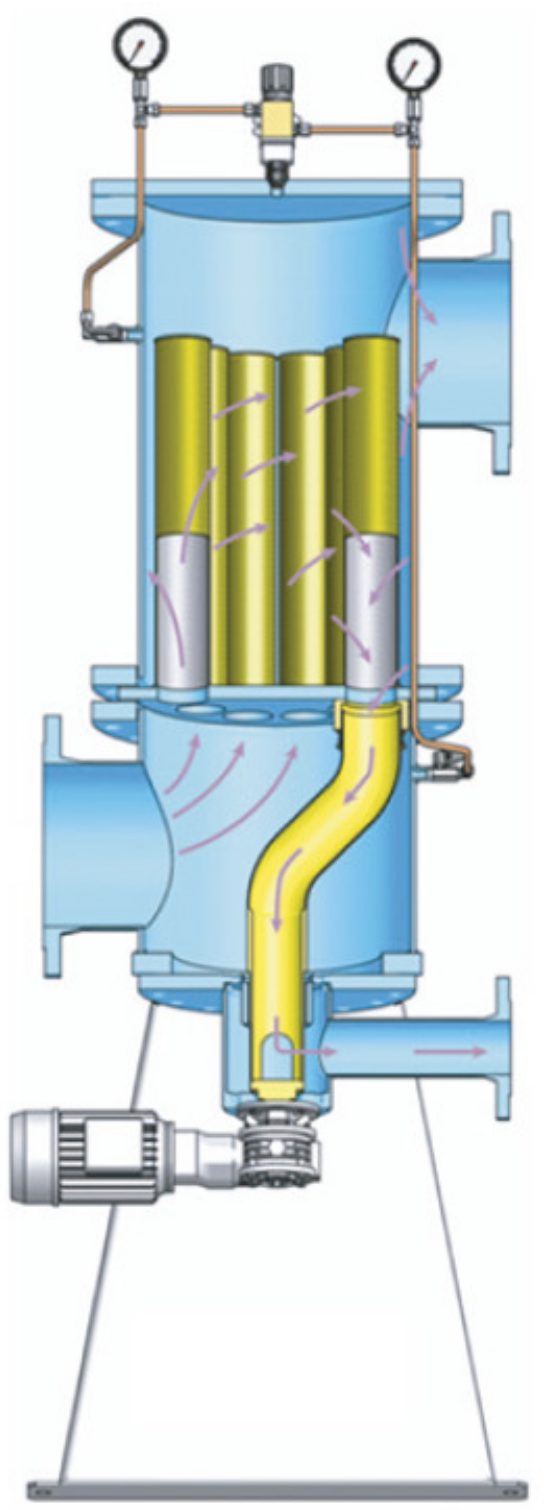

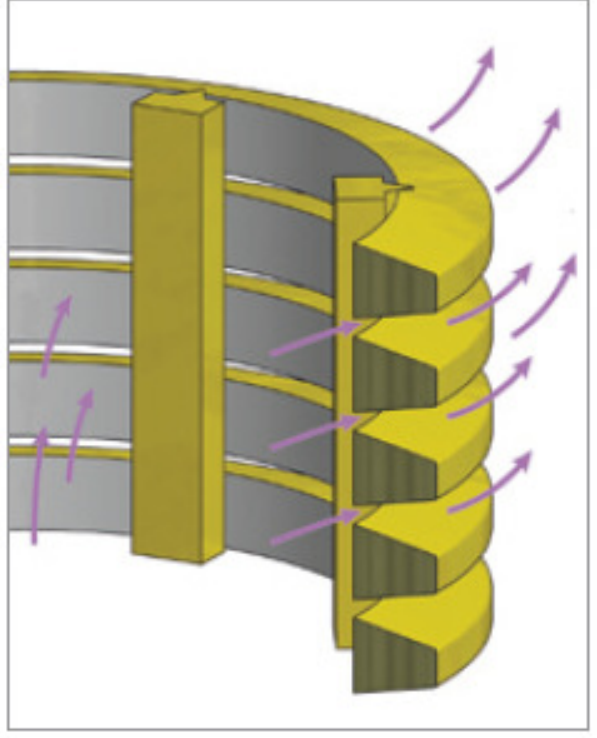

Estado: Filtrando

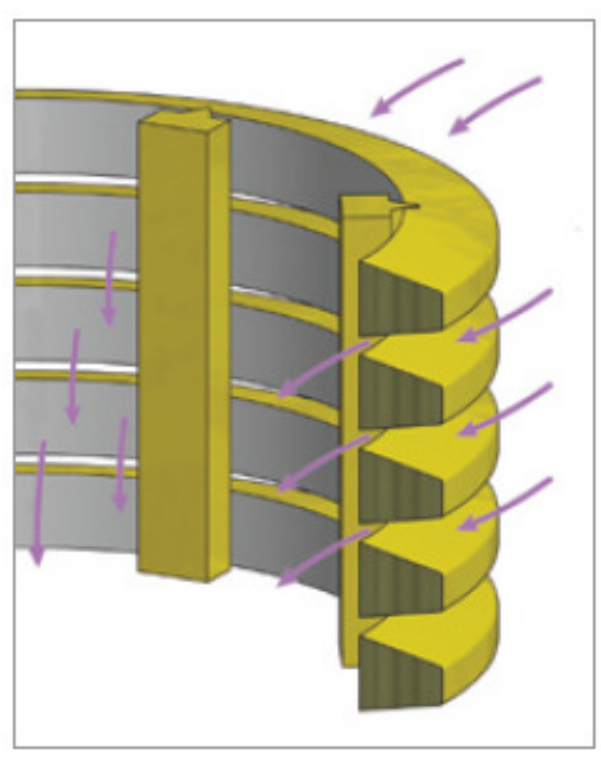

Estado: Limpiando

Principio de funcionamiento

HAWLE. MADE FOR GENERATIONS. 


\section{Dimensiones HAWLE-CANFIL}

HAWLE-CANFIL 100 - 150

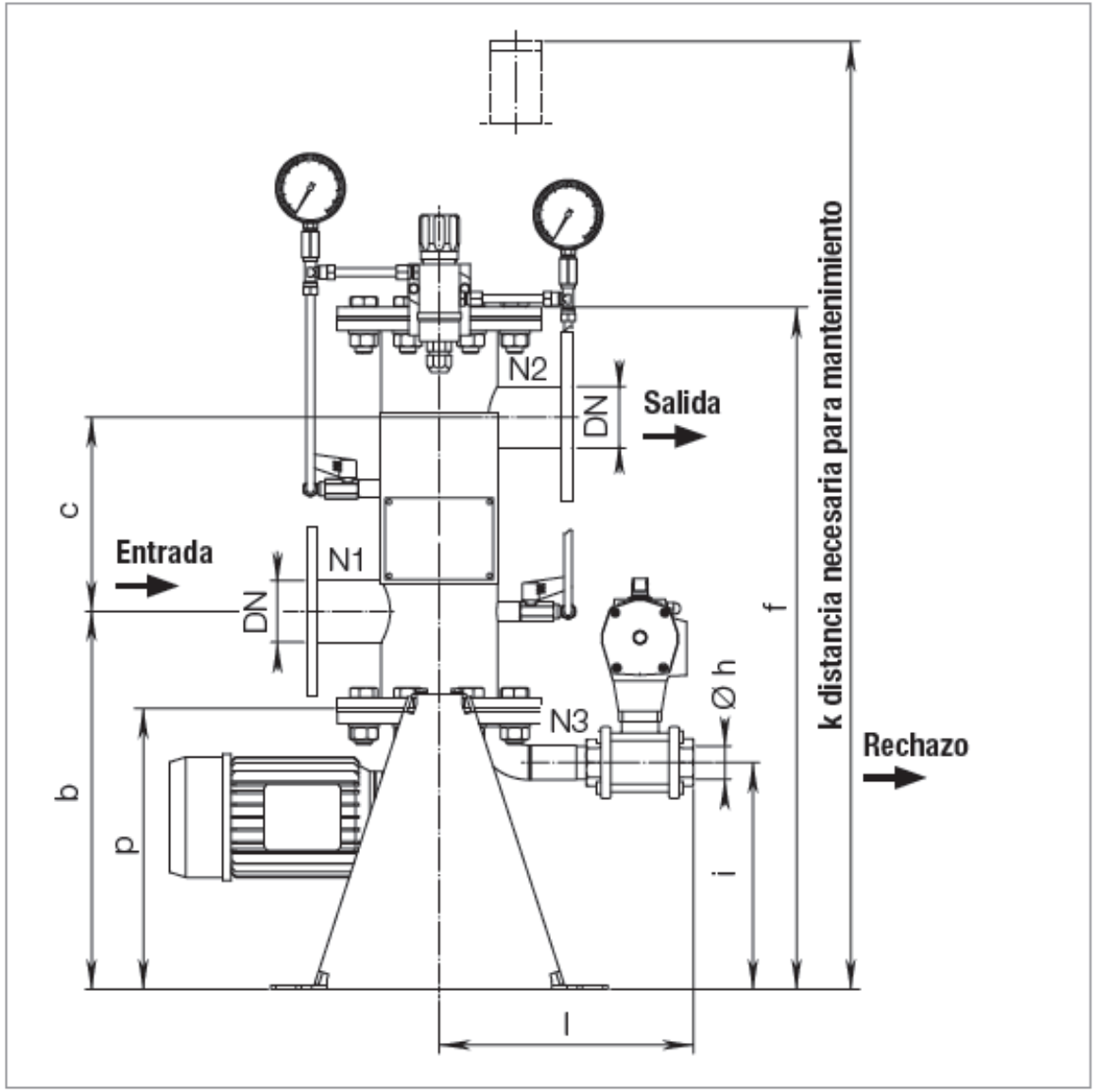

HAWLE-CANFIL 200 - 500

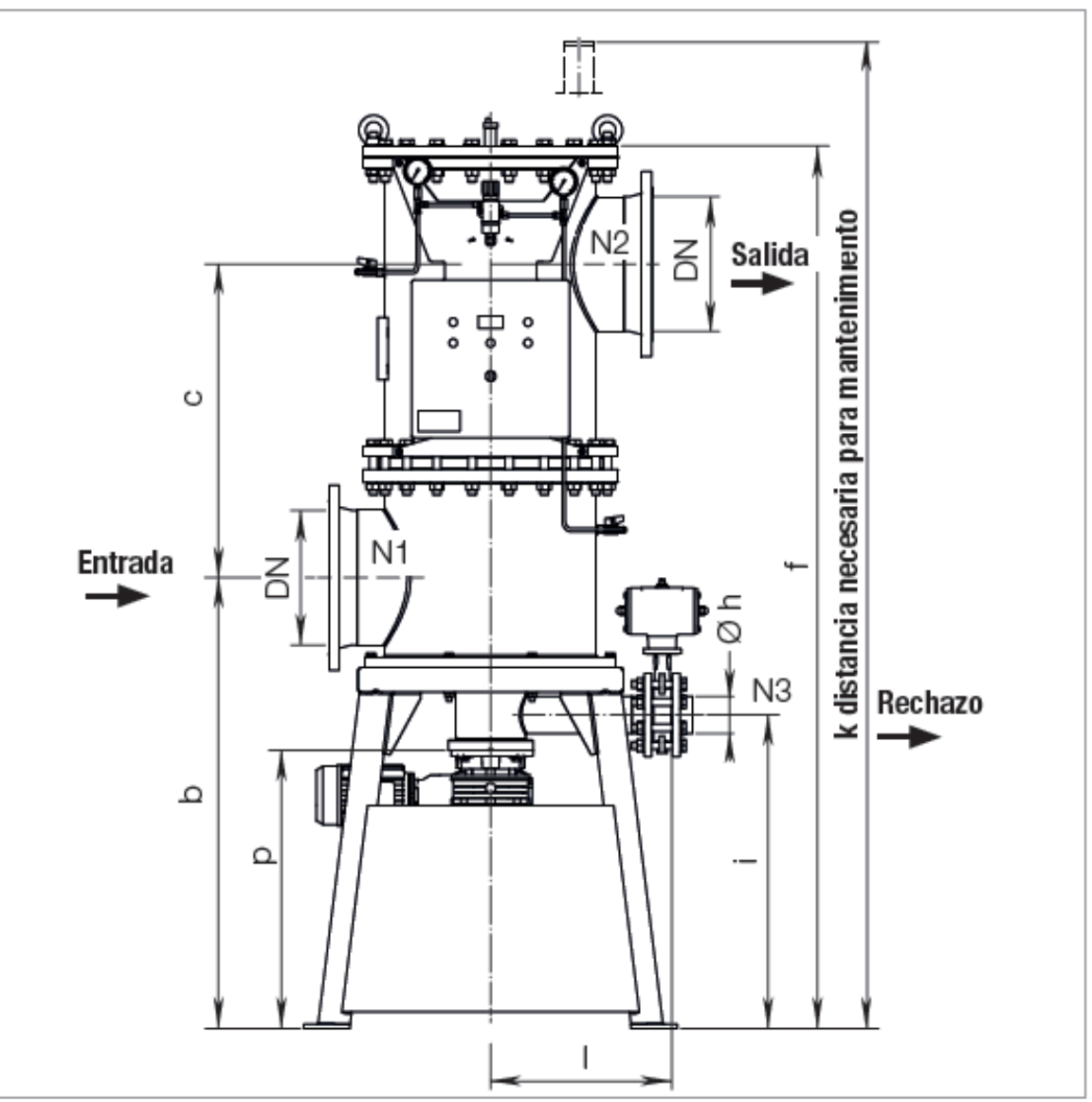




\section{Especificaciones HAWLE-CANFIL}

\begin{tabular}{|l|c|c|c|c|c|c|}
\cline { 2 - 7 } \multicolumn{1}{c|}{} & N1/N2 & \multicolumn{5}{c|}{ Caudal en m3/h } \\
\hline Tipo & DN & $\mathbf{5 0 \mu \mathrm { m }}$ & $\mathbf{7 5 \mu \mathrm { m }}$ & $\mathbf{1 0 0 \mu \mathrm { m }}$ & $\mathbf{1 5 0 \mu \mathrm { m }}$ & $\mathbf{2 0 0 \mu \mathrm { m }}$ \\
\hline CANFIL-100 & 50 & 7 & 10 & 13 & 18 & 20 \\
\hline CANFIL-150 & 80 & 25 & 36 & 47 & 53 & 53 \\
\hline CANFIL-200 & 100 & 55 & 81 & 105 & 115 & 115 \\
\hline CANFIL-300 & 150 & 119 & 174 & 227 & 251 & 251 \\
\hline CANFIL-350 & 200 & 170 & 248 & 324 & 388 & 388 \\
\hline CANFIL-400 & 250 & 279 & 408 & 523 & 523 & 523 \\
\hline CANFIL-500 & 300 & 454 & 665 & 866 & 948 & 948 \\
\hline
\end{tabular}




\section{Referencias HAWLE-CANFIL}

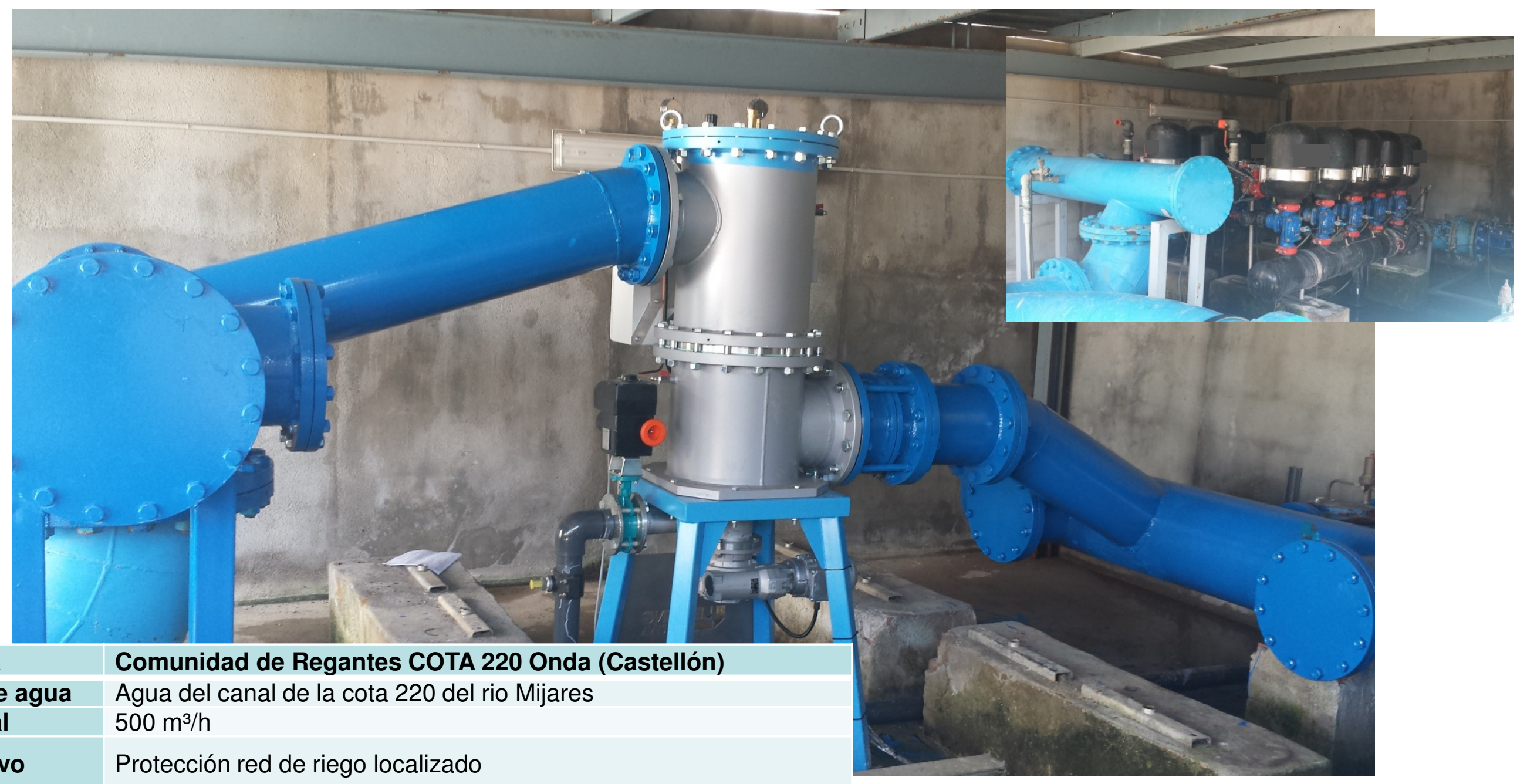

Planta

Fuente agua

Caudal

Objetivo

Condiciones

Solución
- Presión de trabajo 6 bares

- Presión máxima 12 bares (fallo de válvula reductora de presión) HAWLE-CANFIL-400 DN 250, malla de $100 \mu \mathrm{m}$. 


\section{Referencias HAWLE-CANFIL}

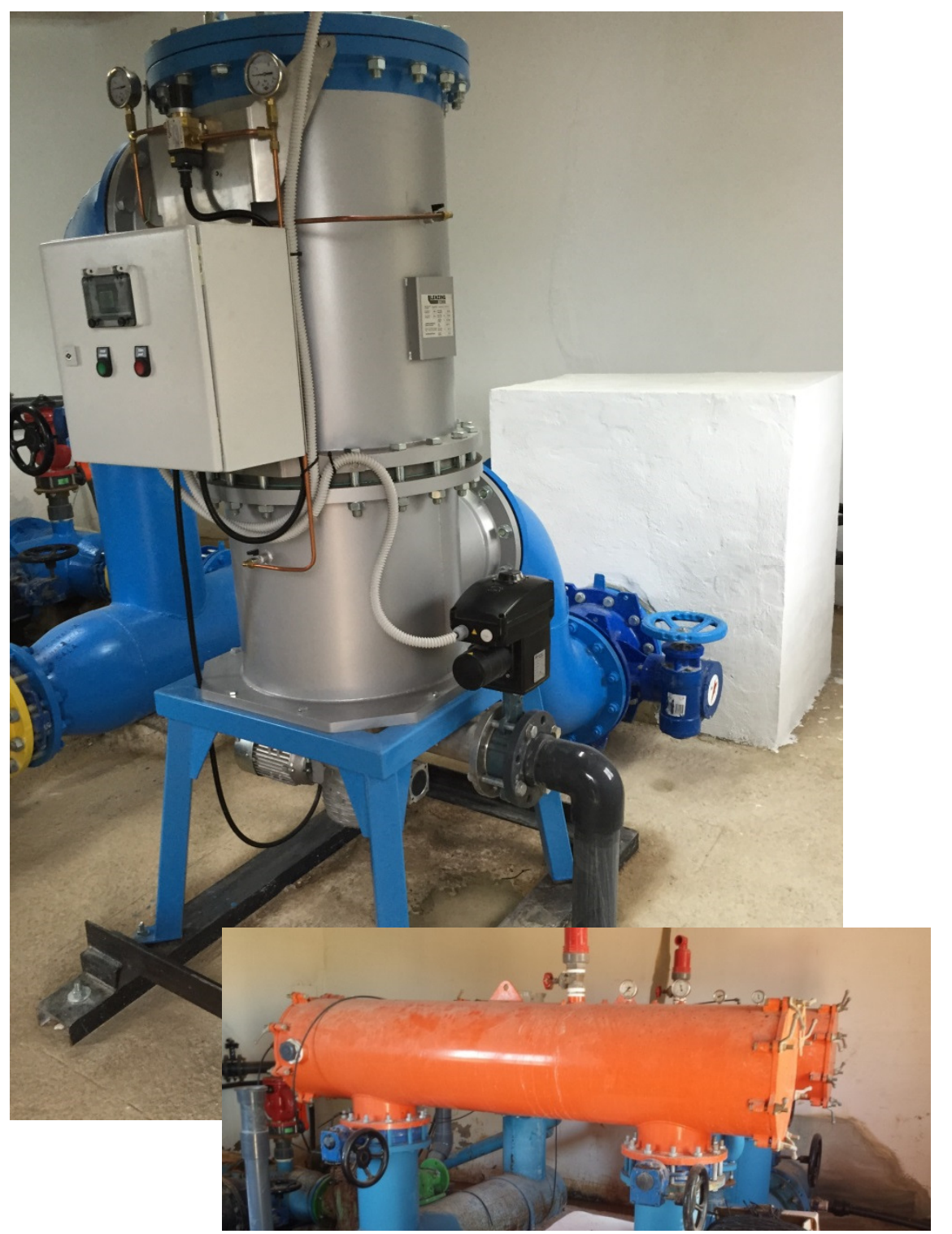

\begin{tabular}{|l|}
\hline Planta \\
\hline Fuente agua \\
\hline Caudal \\
\hline Objetivo \\
\hline Condiciones \\
\hline Solución \\
\hline
\end{tabular}

Comunidad de Regantes Pozo San Juan de Bugarra (Valencia)

Fuente agua Agua del río Turia $800 \mathrm{~m}^{3} / \mathrm{h}$

Protección red de riego localizado

- Presión de trabajo 2,4 bares

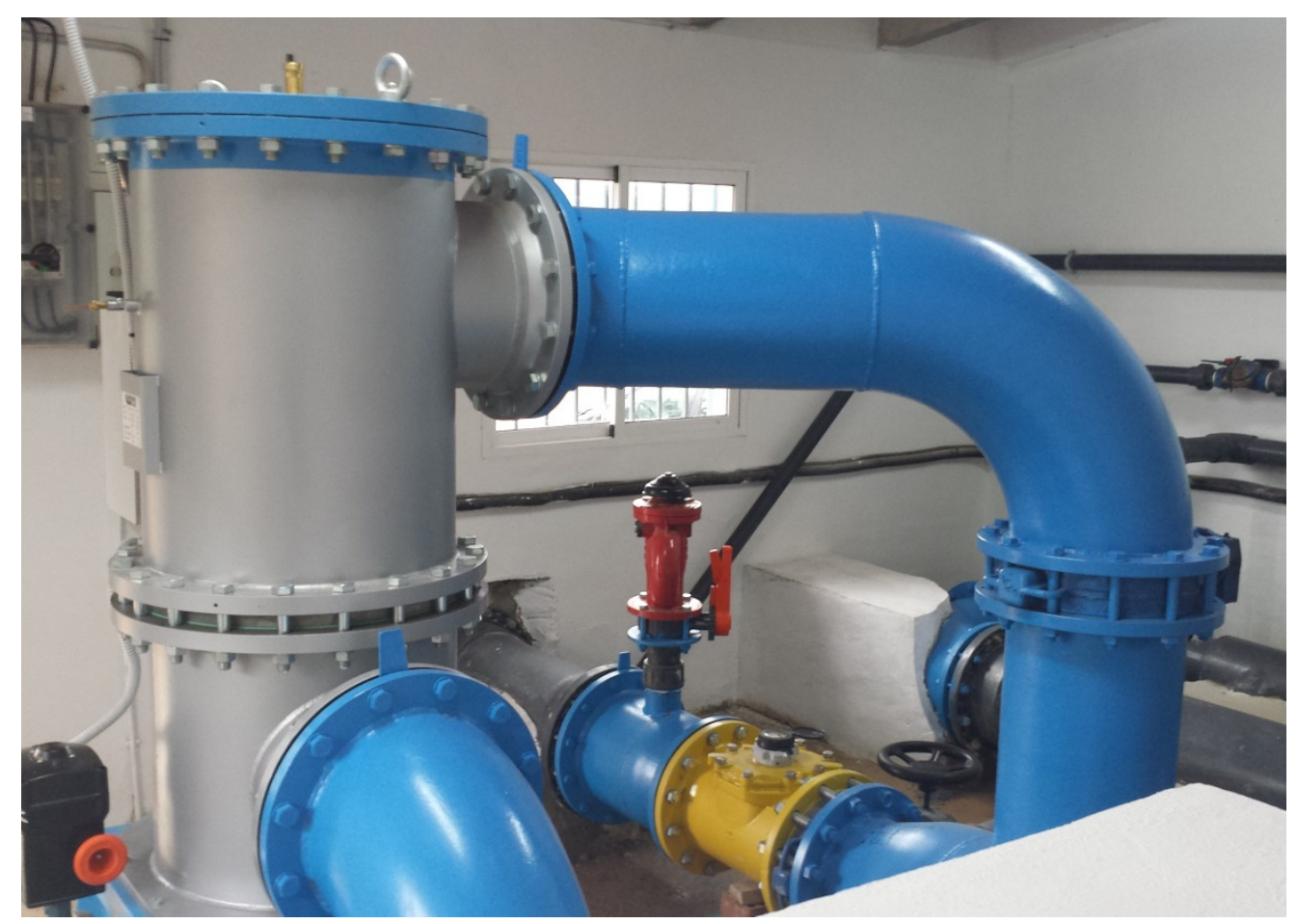

HAWLE. MADE FOR GENERATIONS. 


\section{HEMOS VISTO:}

\section{Visión general del filtrado}

\section{HAWLE-OPTIFIL}

»Visión general de la reutilización

» Simulación de funcionamiento

»Detalles de construcción

»Certificados

»Especificaciones Técnicas

»Referencias

»Planta Móvil de tratamiento de agua

\section{HAWLE-CANFIL}

»Áreas de aplicación

» Funcionamiento

»Dimensiones

»Especificaciones Técnicas

»Referencias 


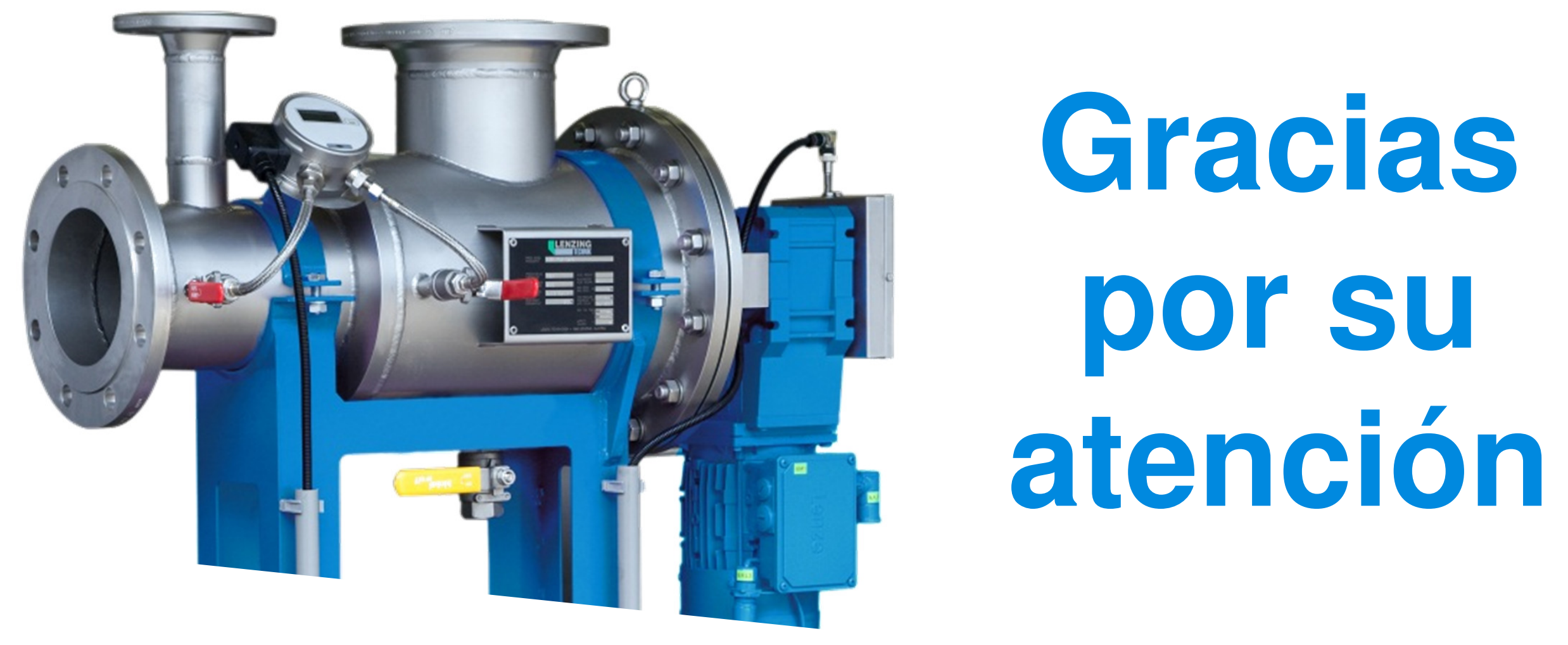

The new spirit of filtration 